\title{
Homotopy decompositions of orbit spaces and the Webb conjecture
}

\author{
by \\ Jolanta Słomińska (Warszawa)
}

\begin{abstract}
Let $p$ be a prime number. We prove that if $G$ is a compact Lie group with a non-trivial $p$-subgroup, then the orbit space $\left(B \mathcal{A}_{p}(G)\right) / G$ of the classifying space of the category associated to the $G$-poset $\mathcal{A}_{p}(G)$ of all non-trivial elementary abelian $p$-subgroups of $G$ is contractible. This gives, for every $G$-CW-complex $X$ each of whose isotropy groups contains a non-trivial $p$-subgroup, a decomposition of $X / G$ as a homotopy colimit of the functor $X^{E_{n}} /\left(N E_{0} \cap \ldots \cap N E_{n}\right)$ defined over the poset $\left(\operatorname{sd} \mathcal{A}_{p}(G)\right) / G$, where sd is the barycentric subdivision. We also investigate some other equivariant homotopy and homology decompositions of $X$ and prove that if $G$ is a compact Lie group with a non-trivial $p$-subgroup, then the map $E G \times_{G} B \mathcal{A}_{p}(G) \rightarrow B G$ induced by the $G$-map $B \mathcal{A}_{p}(G) \rightarrow *$ is a $\bmod p$ homology isomorphism.
\end{abstract}

Introduction. In this paper we will study homotopy and homology decompositions which are associated to the equivariant structure of a $G$-CWcomplex $X$ where $G$ is a Lie group. We will try to generalize and streamline techniques of such decompositions.

Let $\mathcal{C}$ be a small topological category and let $F: \mathcal{C} \rightarrow G$-CW be a functor such that, for every $c \in \mathcal{C}, F(c)=G \times_{H(c)} X^{H^{\prime}(c)}$ where $H(c), H^{\prime}(c)$ are closed subgroups of $G$ and $H(c)$ is a subgroup of the normalizer $N H^{\prime}(c)=$ $N_{G} H^{\prime}(c)$ of $H^{\prime}(c)$ in $G$. Suppose also that there is a natural transformation from $F$ to the constant functor $X$ induced by the inclusions $X^{H^{\prime}(c)} \rightarrow X$. $G$-maps

$$
u: \operatorname{hocolim}_{c \in \mathcal{C}} G \times_{H(c)} X^{H^{\prime}(c)} \rightarrow X
$$

induced by such natural transformations can be used in constructing different homotopy and homology decompositions. If $u$ is a $G$-homotopy equivalence then it will be called a $G$-homotopy decomposition of $X$.

In Section 0 we will introduce a "universal" category $\mathcal{C}_{G}$ and, for every $G$-CW-complex $X$, a functor $\widehat{X}: \mathcal{C}_{G} \rightarrow G$-CW and a natural transformation

2000 Mathematics Subject Classification: 55P91, 55U40, 18G10, 18G40.

Supported by Polish KBN Grants 2 P03A 01113 and 2P03A 002018. 
of functors $\widehat{X} \rightarrow X$. We will study the decompositions induced by functors $F$ which are compositions $\widehat{X} F^{\prime}$, where $F^{\prime}: \mathcal{C} \rightarrow \mathcal{C}_{G}$.

For a given $G$-CW-complex $K$, we will investigate homotopy decompositions of the orbit space $K \times_{G} X$, i.e. homotopy equivalences of the form

$$
\text { id } \times_{G} u: \operatorname{hocolim}_{c \in \mathcal{C}} K \times_{H(c)} X^{H^{\prime}(c)} \simeq K \times_{G} X .
$$

We will also study mod $p$ homology decompositions. In this case the map id $\times_{G} u$ is an $F_{p}$-equivalence. We will show how the known examples of decompositions of $K \times_{G} X$ can be described using $\mathcal{C}_{G}$.

The best known examples of homology decompositions are the cases where $K=E G$ is a universal free $G$-space and $X=*$ is a one-point space ([JM2], [JMO]).

Let $p$ be a prime number and let $\mathcal{A}_{p}(G)$ be the $G$-poset of all elementary abelian non-trivial $p$-subgroups of $G$. If $G$ does not contain a $p$-subgroup, then the set $\mathcal{A}_{p}(G)$ is empty. Let $A_{p}(G)$ be the category whose objects are elements of $\mathcal{A}_{p}(G)$ and whose morphisms are homomorphisms which are restrictions of inner automorphisms of $G$. Let $C_{G}(E)$ be the centralizer of $E$ in $G$. There is a contravariant functor $F: A_{p}(G) \rightarrow G$-CW such that $F(E)=G \times_{C_{G} E} X^{E}$. In the case where $X=*$ and $G$ is a compact Lie group which contains a non-trivial $p$-subgroup, there is a mod $p$ homology decomposition (Theorem 1.3 of [JM2])

$$
\operatorname{hocolim}_{E \in A_{p}(G)} B C_{G}(E) \rightarrow B G \text {. }
$$

Using this fact it is proved in [H1] that if the isotropy groups of $X$ are compact and contain a non-trivial $p$-group, then the map

$$
\operatorname{hocolim}_{E \in A_{p}(G)} E G \times_{C_{G}(E)} X^{E} \rightarrow E G \times_{G} X
$$

is a $\bmod p$ homology isomorphism.

We will prove that one can take instead of $E G$ any $F_{p}$-acyclic complex $K$. We will also construct, for such $K$, another $\bmod p$ homology decomposition

$$
\operatorname{hocolim}_{\left[\left(E_{0}, \ldots, E_{n}\right)\right] \in\left(\operatorname{sd} \mathcal{A}_{p}(G)\right) / G} K \times \times_{N E_{0} \cap \ldots \cap N E_{n}} X^{E_{n}} \rightarrow K \times_{G} X .
$$

Here we take $\mathcal{C}$ equal to the poset $\left(\operatorname{sd} \mathcal{A}_{p}(G)\right) / G$ of the orbits of the $G$-action on the barycentric subdivision of $\mathcal{A}_{p}(G)$. (Recall that the elements of $\operatorname{sd} \mathcal{A}_{p}(G)$ are the increasing sequences $\left(E_{0}<\ldots<E_{n}\right)$ of elements of $\mathcal{A}_{p}(G)$.) If $G$ is a compact Lie group, then in the special case when $X=*$ and $K=E G$, we obtain a $\bmod p$ homology isomorphism

$$
\operatorname{hocolim}_{\left[\left(E_{0}, \ldots, E_{n}\right)\right] \in\left(\operatorname{sd} \mathcal{A}_{p}(G)\right) / G} B\left(N E_{0} \cap \ldots \cap N E_{n}\right) \rightarrow B G,
$$

which is in fact equal to the $\bmod p$ isomorphism

$$
E G \times_{G} B\left(\mathcal{A}_{p}(G)\right) \rightarrow B G .
$$


This last fact is well known in the finite case and can be obtained using 1.3 of [JM2]. The compact case is more complicated because of the topological structure of $\mathcal{A}_{p}(G)$.

If $K=*$ then we obtain not only a homology but also a homotopy decomposition of $X / G$ (Theorem 0.1). In the case when $G$ is a compact Lie group and $X=*$ this means that the space $\left(B \mathcal{A}_{p}(G)\right) / G$ is contractible. For finite groups this was conjectured in [We]. A combinatorial proof of this fact in the finite case was given in [Sy]. Our proof is a generalization of an equivariant approach described for finite groups in [S1].

We will also study $h_{G}^{*}$ decompositions, where $h_{G}^{*}$ is a generalized equivariant cohomology theory, i.e. maps $u$ which induce isomorphisms

$$
h_{G}^{*}(u): h_{G}^{*}(X) \rightarrow h_{G}^{*}\left(\operatorname{hocolim}_{c \in \mathcal{C}} G \times_{H(c)} X^{H^{\prime}(c)}\right) .
$$

We will use the fact that such a decomposition gives a spectral sequence

$$
H^{m}\left(\mathcal{C}, h_{H(-)}^{n}\left(X^{H^{\prime}(-)}\right)\right) \Rightarrow h_{G}^{m+n}(X),
$$

where $h_{H}^{*}(-)=h_{G}^{*}\left(G \times_{H}-\right)$ and $H^{m}(\mathcal{C},-)=\lim _{\mathcal{C}}^{m}(-)=\operatorname{Ext}_{\mathcal{C}}^{m}(\mathbb{Z},-)$ are the cohomology groups of the category $\mathcal{C}$ (Ch. XII of [BK], Section 5 of [DF1]).

0. The main results. Let $G$ be a Lie group. Let $\mathcal{O}_{G}$ be the orbit category of $G$ whose objects are the orbits $G / H$, where $H$ is a closed subgroup of $G$. The morphisms of $\mathcal{O}_{G}$ are the equivariant continuous maps. Every morphism $f: G / H \rightarrow G / H_{1}$ corresponds to a class $[g] \in\left(G / H_{1}\right)^{H}$ such that $f\left(\left[g^{\prime}\right]\right)=g^{\prime} g H_{1}$. It follows from the definitions that $[g] \in\left(G / H_{1}\right)^{H}$ if and only if $H \subseteq g H_{1} g^{-1}$. The topology of the morphism space $\operatorname{Mor}_{\mathcal{O}_{G}}(G / H$, $\left.G / H_{1}\right)=\left(G / H_{1}\right)^{H}$ is induced from $G / H_{1}$. The category $\mathcal{O}_{G}$ is a topological category in the sense of $[\mathrm{HV}]$, i.e. a small category $\mathcal{C}$ with topological morphism sets such that the composition is continuous and the structural map $\mathrm{Ob} \mathcal{C} \rightarrow \operatorname{Mor} \mathcal{C}$ is a closed cofibration. Similarly to $[\mathrm{HV}]$ we will work in the category Top of compactly generated spaces. We will consider $\mathcal{O}_{G}$ as a full subcategory of the category $G$-CW of all $G$-CW-complexes and equivariant cellular maps. This category is described, for example, in [Wi] and [JMO].

We introduce another topological category $\mathcal{C}_{G}$ which plays a crucial role in our considerations concerning equivariant decompositions. Its object set $\mathcal{W}(G)$ consists of all pairs $\left(H, H^{\prime}\right)$ of closed subgroups of $G$ such that $H$ is a subgroup of $N H^{\prime}$. The morphisms $\left(H, H^{\prime}\right) \rightarrow\left(H_{1}, H_{1}^{\prime}\right)$ of $\mathcal{C}_{G}$ are all morphisms $f=[g]: G / H \rightarrow G / H_{1}$ of $\mathcal{O}_{G}$ such that $H_{1}^{\prime} \subseteq g^{-1} H^{\prime} g$. If $f^{\prime}=\left[g^{\prime}\right]:\left(H_{1}, H_{1}^{\prime}\right) \rightarrow\left(H_{2}, H_{2}^{\prime}\right)$ is a morphism of $\mathcal{C}_{G}$, then the condition $H_{2}^{\prime} \subseteq g^{\prime-1} H_{1}^{\prime} g^{\prime}$ implies that $H_{2}^{\prime} \subseteq g^{\prime-1} g^{-1} H^{\prime} g g^{\prime}$ so $f^{\prime} f=\left[g g^{\prime}\right]$ is a morphism of $\mathcal{C}_{G}$. The topology of the morphism spaces is induced from the morphism space topology in $\mathcal{O}_{G}$. There is an inclusion of categories $i: \mathcal{O}_{G} \rightarrow \mathcal{C}_{G}$ such that $i(H)=(H, e)$. The category $\mathcal{C}_{G}$ has a final object $(G, e)$. 
Let $X$ be a $G$-CW-complex. Let $\widehat{X}: \mathcal{C}_{G} \rightarrow G$-CW be the functor defined by $\widehat{X}\left(H, H^{\prime}\right)=G \times_{H} X^{H^{\prime}}, \widehat{X}([g])\left(\left[g^{\prime}, x\right]\right)=\left[g^{\prime} g, g^{-1} x\right]$. Hence $\widehat{X}(G, e)=$ $G \times{ }_{G} X=X$. The equivariant maps

$$
\alpha\left(H, H^{\prime}\right)=\widehat{X}([e]): G \times_{H} X^{H^{\prime}} \rightarrow X
$$

such that $\alpha\left(H, H^{\prime}\right)\left[g^{\prime}, x\right]=g^{\prime} x$ form a natural transformation of functors $\alpha: \widehat{X} \rightarrow X$ where $X$ is the constant functor. Let $\mathcal{C}$ be a topological category. Suppose that we have a functor $\left(H(-), H^{\prime}(-)\right): \mathcal{C} \rightarrow \mathcal{C}_{G}$. Then $\alpha$ induces a G-map

$$
u: \operatorname{hocolim}_{c \in \mathcal{C}} G \times_{H(c)} X^{H^{\prime}(c)} \rightarrow X .
$$

Many examples of decompositions induced by such maps will be described and studied in Sections 3 and 4. For example, let $V$ be a $G$-set of closed subgroups of $G$ and let $\mathcal{O}_{V}$ be the full subcategory of $\mathcal{O}_{G}$ such that $G / H$ is an object of $\mathcal{O}_{V}$ if and only if $H \in V$. Let $\mathcal{C}(V)$ be the full subcategory of $\mathcal{C}_{G}$ whose objects are pairs $\left(H, H^{\prime}\right)$ where $H$ is a subgroup of $H^{\prime}$ and $H^{\prime} \in V$. We will prove the following result in Section 3 .

0.0. Proposition. Assume that all isotropy groups of $X$ are in $V$. Then:

(i) The map

$$
u: \operatorname{hocolim}_{\mathcal{C}(V)} \widehat{X} \rightarrow X
$$

is a G-homotopy decomposition.

(ii) The map $u / G$ gives a homotopy decomposition

$$
\operatorname{hocolim}_{G / H \in \mathcal{O}_{V}} X^{H} \simeq X / G \text {. }
$$

The homotopy decomposition from (ii) is well known. It appears in [E] and [DF2].

In Sections 1 and 2 we will consider the case where $\mathcal{C}$ is the orbit category of the barycentric subdivision of a poset of subgroups of $G$. In order to describe this case we need the following notation. Let $W$ be a topological $G$-poset. This means that $W$ is a topological poset in the sense of [Z̄] (i.e. the order relation is a closed subset of $W^{2}$ ) together with a continuous and order preserving action of $G$ on $W$. Let $d_{n} W$ denote the $G$-subspace of $W^{n+1}$ consisting of all non-decreasing sequences $w .=\left(w_{0}, \ldots, w_{n}\right)$. The $G$-subspace of $d_{n} W$ consisting of all $w$. such that $w_{i} \neq w_{i+1}$ for all $i$ will be denoted by $\operatorname{sd}_{n} W$. The disjoint union $\operatorname{sd} W=\bigsqcup_{n \in \mathbb{N}} \operatorname{sd}_{n} W$ is a topological $G$-poset such that $\left(w_{0}, \ldots, w_{n}\right) \leq\left(w_{0}^{\prime}, \ldots, w_{m}^{\prime}\right)$ if and only if $\left\{w_{0}^{\prime}, \ldots, w_{m}^{\prime}\right\} \subseteq\left\{w_{0}, \ldots, w_{n}\right\}$. There are two $G$-poset maps $p_{0}: \operatorname{sd} W \rightarrow W$ and $p_{1}:(\operatorname{sd} W)^{\mathrm{op}} \rightarrow W$ such that $p_{0}(w)=.w_{0}, p_{1}(w)=.w_{n}$. We will assume that as a topological space, $W$ is equal to the disjoint union of its $G$-orbits $G w=G / G_{w}$ with the topology induced from the topology of $G$. In this case the topological space $W / G$ is discrete. If $W$ satisfies the condition that $w \leq g w$ implies that $w=g w$ then $W / G$ is a poset such that $[w] \leq\left[w^{\prime}\right]$ 
if and only if $w \leq g w^{\prime}$ for some $g \in G$. The $G$-poset $\operatorname{sd} W$ satisfies this condition.

Let $\mathcal{S}(G)$ denote the poset of all closed subgroups of $G$. The group $G$ acts on $\mathcal{S}(G)$ by conjugation. If $H \in \mathcal{S}(G)$, then the isotropy group of this action at $H$ is equal to $N H$. We will assume that $\mathcal{S}(G)$ is a topological space equal to the disjoint union of its $G$-orbits $G x$ with topology induced from the topology of $G$. Let $W$ be a $G$-subposet of $\mathcal{S}(G)$ satisfying the condition that $w \leq g w$ implies that $w=g w$. Suppose that $(\operatorname{sd} W) / G$ is also a discrete space. Then the space sd $W$ is equal to the disjoint union of its $G$-orbits $G /\left(N w_{0} \cap \ldots \cap N w_{n}\right)$. There is a functor $F:(\operatorname{sd} W) / G \rightarrow \mathcal{C}_{G}$ such that

$$
F\left(\left[w_{0}, \ldots, w_{n}\right]\right)=\left(N w_{0} \cap \ldots \cap N w_{n}, w_{n}\right) .
$$

If $\left[w_{0}, \ldots, w_{n}\right] \leq\left[w_{0}^{\prime}, \ldots, w_{m}^{\prime}\right]$, then there exists exactly one element $[g]$ of $G /\left(N w_{0}^{\prime} \cap \ldots \cap N w_{m}^{\prime}\right)$ such that $\left(w_{0}, \ldots, w_{n}\right) \leq\left(g w_{0}^{\prime} g^{-1}, \ldots, g w_{m} g^{-1}\right)$. This implies that $g w_{m}^{\prime} g^{-1} \subseteq w_{n}$ and $F\left(\left[w_{0}, \ldots, w_{n}\right] \leq\left[w_{0}, \ldots, w_{m}^{\prime}\right]\right)$ is the morphism of $\mathcal{C}_{G}$ defined by $[g]$.

If $X$ is a $G$-CW-complex then there is a functor $\tilde{X}:(\operatorname{sd} W) / G \rightarrow G$-CW such that

$$
\widetilde{X}\left(\left[w_{0}, \ldots, w_{n}\right]\right)=G \times_{N w_{0} \cap \ldots \cap N w_{n}} X^{w_{n}} .
$$

In Section 2 of this paper we will prove the following result which in the case when $G$ is a finite group was proved in [S1] (2.10.iv and 2.11).

0.1. Theorem. Let $X$ be a $G$-CW-complex such that all its isotropy groups are compact and contain a non-trivial p-subgroup. Then there is a homotopy equivalence

$$
\operatorname{hocolim}_{\left[\left(E_{0}, \ldots, E_{n}\right)\right] \in\left(\operatorname{sd} \mathcal{A}_{p}(G)\right) / G} X^{E_{n}} /\left(N E_{0} \cap \ldots \cap N E_{n}\right) \simeq X / G .
$$

If $X=*$ is a one-point $G$-CW-complex, then

$$
\widetilde{*}\left(\left[w_{0}, \ldots, w_{n}\right]\right)=G /\left(N w_{0} \cap \ldots \cap N w_{n}\right)
$$

and 0.1 specializes to the fact that, in the case when $G$ is a compact Lie group, the classifying space $B\left(\left(\operatorname{sd} \mathcal{A}_{p}(G)\right) / G\right)$ of the category associated to the poset $\left(\operatorname{sd} \mathcal{A}_{p}(G)\right) / G$ is contractible.

If $W$ is a poset (discrete as topological space), then the geometrical realization $|W|$ of the simplicial complex associated to $W$ is equal to the classifying space $B W$ of the category associated to $W$. An action of $G$ on $W$ induces a $G$-action on $|W|$. Then there are homotopy equivalences $|\operatorname{sd} W| / G \simeq|W| / G$ and $|\operatorname{sd} W| / G \simeq|(\operatorname{sd} W) / G|$. Let $G$ be a finite group. Let $\mathcal{S}_{p}(G)$ be the $G$-poset of all non-trivial $p$-subgroups of $G$. Then the spaces $\left|\mathcal{S}_{p}(G)\right|$ and $\left|\mathcal{A}_{p}(G)\right|$ are $G$-homotopy equivalent (Theorem 2 of [TW]). It is proved in [We] (2.6.1) that $\left|\mathcal{S}_{p}(G)\right| / G$ is $F_{p}$-acyclic and conjectured that $\left|\mathcal{S}_{p}(G)\right| / G$ is contractible. It is also proved in [We] $(2.1 .2)$ that $\left|\mathcal{S}_{p}(G)\right|^{H}$ is 
contractible whenever $H$ is a subgroup of $G$ which contains a normal nontrivial $p$-subgroup. In [S1] a proof of the Webb conjecture was presented which uses this fact and methods introduced in [O1]. We will generalize this proof to the case of a compact Lie group.

If $W$ is a topological poset then the morphism space of the topological category associated to the poset $W$ has topology induced from the topology of $W \times W$ and the classifying space $B W$ of this category is equal to $\bigsqcup_{n \in \mathbb{N}} \Delta_{n} \times d_{n} W / \sim$ where $\Delta_{n}$ is the standard $n$-dimensional simplex and $\sim$ is an appropriate equivalence relation (3.6 of $[\check{Z}]$ ).

Let $W$ be a topological $G$-poset such that the condition that $w \leq g w$ implies that $w=g w$. Then $W / G$ is a topological poset. Suppose that the topological space $W / G$ is discrete and that, for every $n \in \mathbb{N},\left(d_{n} W\right) / G$ is discrete. (This holds for example if $W$ is a subposet of $\mathcal{S}(G)$ and all subgroups in $W$ are finite. Indeed, let $p:\left(d_{n} W\right) / G \rightarrow W / G$ be the projection such that $p\left(\left[w_{0}, \ldots, w_{n}\right]\right)=\left[w_{n}\right]$. Then, for every $[w] \in W / G$, the preimage $p^{-1}([w])$ is a finite space.) The topological space $\operatorname{sd} W / G=(\operatorname{sd} W) / G$ is also discrete in this case and $B W=\bigsqcup_{n \in \mathbb{N}} \Delta_{n} \times \operatorname{sd}_{n} W / \sim$. There is a natural $G$-CW-complex structure on $B W$ such that the poset sd $W / G$ is equal to the poset of the $G$-cells of $B W$. We will show in Section 2 (cf. the proof of 2.3) that

$$
(B W) / G=\bigsqcup_{n \in \mathbb{N}} \Delta_{n} \times\left(\operatorname{sd}_{n} W\right) / G / \sim
$$

is a classifying space $B((\operatorname{sd} W) / G)$ of the category associated to the poset sd $W / G$. We will also show that there are $G$-homotopy equivalences

$$
\operatorname{hocolim}_{\left[\left(w_{0}, \ldots, w_{n}\right)\right] \in \operatorname{sd} W / G} G /\left(N w_{0} \cap \ldots \cap N w_{n}\right) \simeq B \operatorname{sd} W \simeq B W .
$$

In Section 1 we will prove that if $G$ is a compact Lie group and contains a non-trivial $p$-subgroup, then the space $B \mathcal{A}_{p}(G) / G$ is contractible. The proof consists of several steps which will be described below. Recall that $P$ is a p-toral group if its identity component $P_{0}$ is a torus and $\pi_{0}(P)=P / P_{0}$ is a finite $p$-group. The following result is an immediate consequence of 0.1 but in the proof of 0.1 we will use 0.2 in the case when $X$ has finitely many orbit types. We will prove this fact in Section 1.

0.2. Theorem. Let $G$ be a compact Lie group. Let $X$ be a $G$-CWcomplex such that all its isotropy groups contain a non-trivial p-subgroup. Suppose that $X^{P} / H$ is contractible whenever $P$ is a non-trivial p-toral subgroup of $G$ and $H$ is a closed subgroup of the normalizer NP of $P$ in $G$. Then $X / G$ is contractible.

To prove 0.1 we will also need the following result. 
0.3. Proposition. Let $R$ be a commutative ring. Let $X$ and $Y$ be $G$ $\mathrm{CW}$-complexes such that all their isotropy groups are compact and contain a non-trivial p-subgroup. Let $f: X \rightarrow Y$ be a cellular $G$-map of $G$-CWcomplexes. Then:

(i) If, for every compact subgroup $H$ of $G$ containing a non-trivial normal p-toral subgroup, $f^{H}: X^{H} \rightarrow Y^{H}$ is a homotopy equivalence, then so is $f / G: X / G \rightarrow Y / G$.

(ii) If, for every compact subgroup $H$ of $G$ containing a non-trivial normal p-toral subgroup, $f^{H}: X^{H} \rightarrow Y^{H}$ is an $R$-equivalence, then so is $f / G: X / G \rightarrow Y / G$.

If $G$ is a compact Lie group and $Y=*$ then 0.3 is a consequence of 0.2 and the well known decomposition described in 0.0(ii). This result will be proved in Section 1 in the case when $X$ has finitely many orbit types. We will show that the map $B \mathcal{A}_{p}(G) \rightarrow *$ satisfies the assumptions of $0.3(\mathrm{i})$. Hence $B \mathcal{A}_{p}(G) / G$ is contractible and using this we will infer 0.1. We will also prove 0.3 for an arbitrary Lie group $G$.

Let $W$ be a poset of closed subgroups of $G$. In Section 4 we will describe a condition on $W$ which ensures that $h_{G}^{*}(Y) \rightarrow h_{G}^{*}(X)$ is an isomorphism if $X^{H} \rightarrow Y^{H}$ is an $R$-homology isomorphism for all $H \in W$. As an example we will consider the case when

$$
h_{G}^{*}(X)=H^{*}\left(K \times_{G} X, R\right) .
$$

In particular, we will show how $0.3(\mathrm{ii})$ and the results of [JMO] and [JO] concerning the $\bmod p$ decomposition

$$
\operatorname{hocolim}_{G / P \in \mathcal{O}_{R_{p}(G)}} B P \rightarrow B G,
$$

where $R_{p}(G)$ is a certain poset of $p$-toral subgroups of $G$, imply the following result.

0.4. Proposition. Let $X$ and $Y$ be $G$-CW-complexes such that all their isotropy groups are compact and contain non-trivial p-subgroups. Let $K$ be an $F_{p}$-acyclic $G$-CW-complex. If, for every non-trivial $p$-toral subgroup $H$ of $G, f^{H}: X^{H} \rightarrow Y^{H}$ is an $F_{p}$-equivalence, then so is $\operatorname{id}_{K} \times{ }_{G} f: K \times_{G} X \rightarrow$ $K \times_{G} Y$.

If $G$ is a compact Lie group with a non-trivial $p$-subgroup, then from the fact (cf. the proof of 1.5) that all isotropy groups of $B \mathcal{A}_{p}(G)$ contain nontrivial normal $p$-subgroups and that, for every subgroup $H$ of $G$ containing a non-trivial normal $p$-subgroup, the space $B \mathcal{A}_{p}(G)^{H}$ is contractible, we obtain the following result.

0.5. Corollary. Let $G$ be a compact Lie group with a non-trivial psubgroup. Then the map $E G \times_{G} B \mathcal{A}_{p}(G) \rightarrow B G$ induced by the $G$-map $B \mathcal{A}_{p}(G) \rightarrow *$ is an $F_{p}$-equivalence. 
The following posets of subgroups will be defined and used in the paper.

List of posets of subgroups of $G$

- $\mathcal{A}_{p}(G)$ - the set of all elementary abelian non-trivial $p$-subgroups,

- $\mathcal{A}_{p}^{\prime}(G)$ - the set of all elementary abelian $p$-subgroups,

- $\mathcal{K}_{p}(G)$ - the set of all compact subgroups $H$ such that, for every $P \in \mathcal{M}_{p}(G), H \cap Z(P)$ contains a non-trivial $p$-subgroup,

- $\mathcal{M}_{p}(G)$ - the set of all maximal non-trivial $p$-toral subgroups,

- $\mathcal{N}_{p}(G)$ - the set of all compact subgroups containing a non-trivial normal $p$-toral subgroup,

- $\mathcal{S}(G)$ - the set of all closed subgroups,

- $\mathcal{S}(G, X)$ - the set of all isotropy groups of $X$,

- $\mathcal{S}_{0}(G, X)=\mathcal{S}(G, X) \cup \mathcal{S}(G, *)$,

- $\mathcal{S}_{\mathrm{c}}^{\prime}(G)$ - the set of all compact subgroups,

- $\mathcal{S}_{\mathrm{c}}(G)$ - the set of all compact subgroups which contain a non-trivial $p$-subgroup,

- $\mathcal{S}_{p}^{\prime}(G)$ - the set of all subtoral $p$-subgroups,

- $\mathcal{S}_{p}(G)$ - the set of all subtoral $p$-subgroups which contain a non-trivial $p$-subgroup,

- $\mathcal{T}_{p}^{\prime}(G)$ - the set of all $p$-toral subgroups,

- $\mathcal{T}_{p}(G)$ - the set of all non-trivial $p$-toral subgroups,

- $\mathcal{T}_{p}(G, X)$ - the set of all maximal $p$-toral subgroups of isotropy groups of $X$,

- $\mathcal{Z}_{p}(G)$ - the set of all compact subgroups containing a non-trivial central $p$-subgroup.

1. Orbit spaces of compact Lie group actions. Let $G$ be a Lie group. The set of all compact subgroups of $G$ will be denoted by $\mathcal{S}_{\mathrm{c}}^{\prime}(G)$. The set of all elements of $\mathcal{S}_{\mathrm{c}}^{\prime}(G)$ which contain a non-trivial $p$-subgroup will be denoted by $\mathcal{S}_{\mathrm{c}}(G)$. The set of all closed $p$-toral subgroups of $G$ will be denoted by $\mathcal{T}_{p}^{\prime}(G)$. The set of all non-trivial $p$-toral subgroups of $G$ will be denoted by $\mathcal{T}_{p}(G)$. The set of all compact subgroups of $G$ containing a non-trivial normal $p$-toral subgroup will be denoted by $\mathcal{N}_{p}(G)$.

If $G$ is a compact Lie group, $T$ is a maximal torus of $G$ and $N_{p} T / T$ is a Sylow $p$-subgroup of $N T / T$, then $N_{p} T$ is a maximal $p$-toral subgroup of $G$. All maximal $p$-toral subgroups of $G$ are conjugate to $N_{p} T$ (Lemma A.1 of [JMO]). The set of all maximal $p$-toral subgroups of $G$ will be denoted by $\mathcal{M}_{p}(G)$ and the set of all maximal $p$-toral subgroups of isotropy groups of $X$ by $\mathcal{T}_{p}(G, X)$.

Let $\mathcal{S}$ be a subset of the set of compact subgroups of $G$. We will use the notation

$$
\mathcal{W}_{\mathcal{S}}=\left\{\left(H, H^{\prime}\right): H^{\prime} \subseteq H \subseteq N H^{\prime}, H^{\prime} \in \mathcal{S}, H \in \mathcal{S}_{\mathrm{c}}^{\prime}(G) .\right\}
$$


A non-empty $G$-poset $\mathcal{P}$ of $p$-toral subgroups of $G$ will be called concave if, for any $p$-toral subgroups $P$ and $P^{\prime}$ the condition that $P \subseteq P^{\prime}$ and $P \in \mathcal{P}$ implies that $P^{\prime} \in \mathcal{P}$. If $G$ is a compact Lie group and $\mathcal{P}$ is concave, then $\mathcal{M}_{p}(G) \subseteq \mathcal{P}$ because all maximal $p$-toral subgroups are conjugate by elements of $G$.

Let $\mathcal{C W}$ denote the category of spaces having the homotopy type of $\mathrm{CW}$-complexes and let $\mathcal{C W}_{0}$ be the subcategory of $\mathcal{C W}$ consisting of the connected spaces. We will say that a class $\mathcal{A}$ of objects of $\mathcal{C W}$ is thick if it is closed under homotopy equivalences and taking homotopy pushouts.

In this section we will assume that $G$ is a compact Lie group with a non-trivial $p$-subgroup and that $X$ is a $G$-CW-complex with finitely many orbit types.

1.1. Theorem. Let $\mathcal{A}$ be thick. Let $\mathcal{P}$ be a concave $G$-poset of p-toral subgroups of $G$ containing all maximal p-toral subgroups of the isotropy groups of $X$. If $X^{P} / H \in \mathcal{A}$ whenever $P \in \mathcal{P}$ and $P \subseteq H \subseteq N P$, then $X / G \in \mathcal{A}$.

Proof. If $(e) \in \mathcal{P}$, then the assumptions imply that $X / G \in \mathcal{A}$. Let $k(G, X)$ denote the number of elements of $\mathcal{T}_{p}(G, X) / G$.

If $k(G, X)=1$, then $\mathcal{T}_{p}(G, X)=(P)=\left\{g P g^{-1}: g \in G\right\}$, where $P$ is, up to conjugacy, the unique maximal $p$-toral group of an isotropy group of $X$. Hence $X=X^{(P)}=\bigcup_{P^{\prime} \in(P)} X^{P^{\prime}}$. It is proved in [O1] (in the proof of Proposition 3) that the map $X^{P} / N P \rightarrow X^{(P)} / G$ is a homeomorphism. (This is a consequence of the fact that, if $G^{\prime}$ is a closed subgroup of $G$ and $P$ is a maximal $p$-toral subgroup of $G^{\prime}$, then $N P$ acts transitively on $\left(G / G^{\prime}\right)^{P}$. Indeed, let $a G^{\prime}, b G^{\prime} \in\left(G / G^{\prime}\right)^{P}$. Then $a^{-1} P a, b^{-1} P b$ are maximal $p$-toral subgroups of $G^{\prime}$ so they are conjugate in $G^{\prime}$ and there is $c \in G^{\prime}$ such that $b c a^{-1} \in N P$.) If the assumptions hold, then $P$ is a maximal toral $p$-subgroup of $G$. Hence, in this case, $X / G=X^{P} / N P \in \mathcal{A}$.

We use induction on the dimension of $G$ and then on the order of $\pi_{0}(G)=G / G_{0}$, where $G_{0}$ is the identity component of $G$. Assume that the result is true for all proper closed Lie subgroups of $G$. Now we use induction on $k(G, X)$. Let $k(G, X)=k+1>1$. Suppose that the result is true for all $G$-CW-complexes $X^{\prime}$ such that $k\left(G, X^{\prime}\right) \leq k$. Let $P$ be a minimal element of $\mathcal{T}_{p}(G, X)$. As $P$ is not a maximal $p$-toral group, it follows that $N P / P$ contains a non-trivial $p$-toral subgroup (cf. [O1], Lemma 2). Let $X^{\prime}$ be a $G$-CW-subcomplex of $X$ such that $x \in X \backslash X^{\prime}$ if and only if maximal $p$-toral subgroups of the isotropy group $G_{x}$ are conjugate to $P$. The induction assumption implies that $X^{\prime} / G \in \mathcal{A}$ because $k\left(G, X^{\prime}\right) \leq k$. Indeed, let $\mathcal{P}_{o}=\mathcal{P} \backslash(P)$. Then, for every $\left(H, P^{\prime}\right) \in \mathcal{W}_{\mathcal{P}_{o}}$, $X^{P^{\prime}} / H=X^{P^{\prime}} / H$. 
It follows from the definition that $X=X^{\prime} \cup X^{(P)}$ and that $X / G$ is equal to the pushout of the diagram

$$
X^{(P)} / G \leftarrow X^{\prime(P)} / G \rightarrow X^{\prime} / G .
$$

If $x \in X \backslash X^{\prime}$, then $\mathcal{M}_{p}\left(G_{x}\right)$ is a subset of $(P)$ and $N P$ acts transitively on $(G x)^{P}=\left(G / G_{x}\right)^{P}$. Hence $X / G$ is the pushout of the diagram

$$
X^{P} / N P \leftarrow X^{\prime P} / N P \rightarrow X^{\prime} / G .
$$

Since $X^{\prime P} / N P \rightarrow X^{P} / N P$ is a cofibration, $X / G$ is the homotopy pushout of this diagram.

The space $X^{\prime P}$, which has the structure of an $N P$-CW complex, satisfies the assumptions of the proposition. It is of finite orbit type because, for every closed subgroup $G^{\prime}$ of $G,\left(G / G^{\prime}\right)^{P} / N P$ is finite (II.5.7 of [Br1]). Let $\mathcal{P}^{\prime}=\left\{P^{\prime} \in \mathcal{P}: P \subset P^{\prime} \subseteq N P, P^{\prime} \neq P\right\}$. From the fact that, for every $x \in X^{\prime P}, P \subseteq G_{x} \cap N P$ and $P$ is not a maximal $p$-toral subgroup of $G_{x}$, it follows that $P$ is not a maximal $p$-toral subgroup of $G_{x} \cap N P$ (Lemma 2 of [O1]). Hence

$$
\mathcal{T}_{p}\left(N P, X^{\prime P}\right)=\bigcup_{x \in X^{\prime}} \mathcal{M}_{p}\left(G_{x} \cap N P\right) \subseteq \mathcal{P}^{\prime}
$$

and $X^{\prime P^{\prime}} / H=X^{P^{\prime}} / H \in \mathcal{A}$ whenever $\left(H, P^{\prime}\right) \in \mathcal{W}_{\mathcal{P}^{\prime}}$.

If $P$ is a normal subgroup of $G$, then $N P=G$ but $k\left(X^{\prime P}, G\right) \leq k$, because $P \notin \mathcal{T}_{p}\left(G, X^{\prime P}\right) \subseteq \mathcal{T}_{p}(G, X)$. If $P$ is not a normal subgroup of $G$, then $N P<G$ and we can use the induction assumption. In both cases we find that $X^{\prime P} / N P \in \mathcal{A}$. Hence $X / G \in \mathcal{A}$.

In particular, if $\mathcal{P}=\mathcal{T}_{p}(G)$ and $\mathcal{A}$ is the class of all contractible objects of $\mathcal{C} \mathcal{W}_{0}$ then 1.1 specializes to 0.2 .

In what follows let $\mathcal{A}$ be a thick category. We now define three conditions for thick categories.

A1: For every compact Lie group $H$ and for every $H$-CW-complex $X$, if $X^{H^{\prime}} \in \mathcal{A}$ for every closed subgroup $H^{\prime}$ of $H$, then $X / H \in \mathcal{A}$.

A2: For every compact Lie group $H$ and for every $H$-CW-complex $X$, if $\operatorname{dim} X<\infty$ and $X \in \mathcal{A}$, then $X / H \in \mathcal{A}$.

A3: For every compact Lie group $H$ and for every $H$-CW-complex $X$, if $X / P \in \mathcal{A}$ for every $P \in \mathcal{M}_{p}(H)$, then $X / H \in \mathcal{A}$.

Let $H^{\prime}$ be a closed subgroup of $G$ and let $\mathcal{P}$ be a set of subgroups of $G$. We use the notation 


$$
\begin{aligned}
\mathcal{N}_{H^{\prime}} & =\left\{H \in \mathcal{S}(G): H^{\prime} \subseteq H \subseteq N H^{\prime}\right\}, \\
\mathcal{N}_{\mathcal{P}} & =\left\{H \in \mathcal{S}(G): H^{\prime} \subseteq H \subseteq N H^{\prime}, H^{\prime} \in \mathcal{P}\right\}, \\
\mathcal{S}_{\mathcal{P}} & =\bigcup_{P, P^{\prime} \in \mathcal{P}}\left\{H \in \mathcal{S}(G): P \subseteq H \subseteq P^{\prime} \subseteq N P\right\}, \\
\mathcal{S}_{p}^{\prime}(G) & =\mathcal{S}_{\mathcal{T}_{p}^{\prime}(G)}, \quad \mathcal{S}_{p}(G)=\mathcal{S}_{\mathcal{T}_{p}(G)} .
\end{aligned}
$$

1.2. Corollary. Let $\mathcal{P}$ be a concave $G$-poset of p-toral subgroups of $G$. Let $X$ be a $G$-CW-complex such that maximal p-toral subgroups of isotropy groups of $X$ are in $\mathcal{P}$. Suppose that $\mathcal{A}$ is thick and that one of the following conditions holds:

(i) $\mathcal{A}$ satisfies $\mathbf{A} 3$ and $X^{P} / P^{\prime} \in \mathcal{A}$ whenever $\left(P^{\prime}, P\right) \in \mathcal{W}_{\mathcal{P}}$ and $P^{\prime} \in \mathcal{P}$.

(ii) $\mathcal{A}$ satisfies $\mathbf{A} 1$ and $X^{H} \in \mathcal{A}$ whenever $H \in \mathcal{N}_{\mathcal{P}}$.

(iii) $\mathcal{A}$ satisfies $\mathbf{A} 1$ and $\mathbf{A} 3$ and $X^{H} \in \mathcal{A}$ whenever $H \in \mathcal{S}_{\mathcal{P}}$.

(iv) $\mathcal{A}$ satisfies $\mathbf{A 2}, \operatorname{dim} X<\infty$ and $X^{H} \in \mathcal{A}$ whenever $H \in \mathcal{P}$.

Then $X / G \in \mathcal{A}$.

Proof. The result is a consequence of 1.1. Suppose that $\left(H, P^{\prime}\right) \in \mathcal{W}_{\mathcal{P}}$.

If (i) holds, then $X^{P^{\prime}} / P^{\prime \prime} \in \mathcal{A}$ whenever $P^{\prime \prime} \in \mathcal{M}_{p}(H)$. Since $\mathcal{A}$ satisfies A3, it follows that $X^{P^{\prime}} / H \in \mathcal{A}$.

Assume that (ii) holds. Let $H^{\prime}=H / P^{\prime}$ and let $Y=X^{P^{\prime}}$. We can consider $Y$ as an $H^{\prime}$-CW-complex. If $H_{0}^{\prime}$ is a subgroup of $H^{\prime}$, then $H_{0}^{\prime}=$ $H_{0} / P^{\prime}$, where $P^{\prime} \subseteq H_{0} \subseteq H$, and $Y^{H_{0}^{\prime}}=X^{H_{0}} \in \mathcal{A}$ because $H_{0} \in \mathcal{N}_{\mathcal{P}}$. Hence $X^{P^{\prime}} / H=Y / H^{\prime} \in \mathcal{A}$.

If $\mathcal{A}$ satisfies $\mathbf{A} \mathbf{1}$ and $X^{G^{\prime}} \in \mathcal{A}$ whenever $G^{\prime} \in \mathcal{S}_{\mathcal{P}}$ then $X^{P} / P^{\prime} \in \mathcal{A}$ whenever $P, P^{\prime} \in \mathcal{P}, P^{\prime} \in \mathcal{N}_{P}$. Now we can use part (ii) of this result to obtain (iii).

If (iv) holds, then $X^{P^{\prime}} / H \in \mathcal{A}$ by the definitions.

1.3. EXAMPLES. Let

$$
\begin{aligned}
\mathcal{C} & =\left\{X \in \mathcal{C} \mathcal{W}_{0}: X \text { is contractible }\right\} \\
\mathcal{D}(R) & =\left\{X \in \mathcal{C} \mathcal{W}_{0}: X \text { is } R \text {-acyclic }\right\} \\
B_{k}(R) & =\left\{X \in \mathcal{C} \mathcal{W}_{0}: H^{i}(X, R)=0 \text { for } i=1, \ldots, k\right\} .
\end{aligned}
$$

(i) The well known decomposition from 0.0(ii) implies that all these classes satisfy A1.

(ii) The classes $\mathcal{D}\left(F_{p}\right)$ and $B_{k}\left(F_{p}\right)$ satisfy $\mathbf{A 3}$. This is a consequence of the existence of an appropriate transfer. Let $H$ be a closed subgroup of $G$ and let $\pi_{X}: X / H \rightarrow X / G$ be the projection to the orbit space. It is proved in $[\mathrm{O} 2],[\mathrm{LMM}],[\mathrm{LMS}]$ that there exists a natural transfer map

$$
t_{X}: H^{*}(X / H, R) \rightarrow H^{*}(X / G, R)
$$


such that the composition $H^{*}\left(\pi_{X}\right) t_{X}$ is the multiplication by the Euler characteristic $\chi(G / H)$ of $G / H$. If $H$ is a maximal $p$-toral subgroup of $G$, then $\chi(G / H)$ is prime to $p$. Hence, if $H^{n}\left(X / H, F_{p}\right)=0$, then $H^{n}\left(X / G, F_{p}\right)=0$.

(iii) The classes $\mathcal{D}(\mathbb{Z})$ and $\mathcal{D}\left(F_{p}\right)$ satisfy $\mathbf{A 2}$. This follows from Theorems 1 and 2 of [O1].

The next result describes the case when $\mathcal{P}=\mathcal{T}_{p}(G)$ and $\mathcal{A}$ is one of the classes from 1.3. The statement (i) is a special case of 0.3. For a finite group $G$, this result is proved in 2.11 of [S1]. The statement (iii), for finite groups, finite $G$-CW-complexes and $F_{p}$-acyclic spaces, is proved in [We].

1.4. Proposition. Let $X$ be a $G$-CW-complex such that all its isotropy groups contain a non-trivial p-subgroup. Then:

(i) $X / G$ is contractible (resp. $R$-acyclic) if $X^{H}$ is contractible (resp. $R$ acyclic) for all closed subgroups $H$ containing a non-trivial normal $p$-toral subgroup.

(ii) $X / G$ is $F_{p}$-acyclic if, for every $H \in \mathcal{S}_{p}(G), X^{H}$ is $F_{p}$-acyclic.

(iii) If $\operatorname{dim} X<\infty$ and, for every non-trivial p-toral subgroup $H$ of $G$, $X^{H}$ is $\mathbb{Z}$-acyclic (resp. $F_{p}$-acyclic), then $X / G$ is $\mathbb{Z}$-acyclic (resp. $F_{p}$-acyclic).

Proof. $\mathcal{T}_{p}(G)$ is a concave set of $p$-subgroups of $G$. By 1.2(ii), $\mathcal{N}_{\mathcal{T}_{p}(G)}=$ $\mathcal{N}_{p}(G)$ so (i) follows. The statement (ii) is a consequence of 1.2 (iii) because $\mathcal{S}_{\mathcal{T}_{p}(G)}=\mathcal{S}_{p}(G)$, and (iii) follows from 1.2(iv).

1.5. Corollary. If $G$ is a compact Lie group with a non-trivial psubgroup, then the space $B \mathcal{A}_{p}(G) / G$ is contractible.

Proof. It is proved in 6.1 of [JM2] that there are only finitely many conjugacy classes of elementary abelian $p$-subgroups in $G$. If $x \in B \mathcal{A}_{p}(G)$, then $G_{x}=N E_{0} \cap \ldots \cap N E_{k}$, where $E_{i} \in \mathcal{A}_{p}(G)$ and $E_{0}<\ldots<E_{k}$, so $E_{0} \subseteq$ $G_{x} \subseteq N E_{0}$. For every $H \in \mathcal{N}_{p}(G)$, the space $\left(B \mathcal{A}_{p}(G)\right)^{H}=B\left(\mathcal{A}_{p}(G)^{H}\right)$ is contractible. For $G$ finite this follows from 2.1.2 of [We]. The proof for any compact Lie group is similar. The space $\mathcal{A}_{p}(G)^{H}$ is a disjoint union of its $\mathrm{NH} / \mathrm{H}$-orbits. Let

$$
\mathcal{A}_{p}(G)_{\geq E}=\left\{E^{\prime} \in \mathcal{A}_{p}(G): E \subseteq E^{\prime}\right\} .
$$

There exists a non-trivial normal $p$-toral subgroup $P$ of $H$ such that $N H$ is a subgroup of $N P$. Indeed, let $Q$ be the intersection of all maximal $p$-toral subgroups of $H$. Then $N H$ is a subgroup of $N Q$. Let $Q_{0}$ be the component of the identity of $Q$. We can take $P=Q_{0}$ if $Q_{0}$ is non-trivial. If $Q_{0}=e$, then we can take as $P$ the intersection of all Sylow $p$-subgroups of $Q$. In this case $P$ is the maximal normal $p$-toral subgroup of $H$. It follows from A3 of [JMO] and 7.6 of [JM1] that if $P^{\prime} \in \mathcal{T}_{p}(G)$, then the center $Z\left(P^{\prime}\right)$ of $P^{\prime}$ is also in $\mathcal{T}_{p}(G)$. Let $E$ be the maximal elementary abelian $p$-subgroup of $Z(P)$. Then $E \subset H \subset N H \subset N E, N H \subset N C E$ and, for every $E^{\prime} \in \mathcal{A}_{p}(G)^{H}$, 
$E^{\prime} \cap C E=E^{\prime E}$ is a non-trivial group. The poset map $h_{E}: \mathcal{A}_{p}(G)^{H} \rightarrow$ $\left(\mathcal{A}_{p}(G)^{H} \cap \mathcal{A}_{p}(C E)\right)_{\geq E}$ such that $h_{E}\left(E^{\prime}\right)=\left(E^{\prime} \cap C E\right) E$ whenever $E^{\prime} \in$ $\mathcal{A}_{p}(G)^{H}$, is continuous because it is an $N H / H$-poset map. The map $B h_{E}$ is the composition of the homotopy equivalences $B \mathcal{A}_{p}(G)^{H} \rightarrow B\left(\mathcal{A}_{p}(G)^{H} \cap\right.$ $\left.\mathcal{A}_{p}(C E)\right)$ and $B\left(\mathcal{A}_{p}(G)^{H} \cap \mathcal{A}_{p}(C E)\right) \rightarrow B\left(\left(\mathcal{A}_{p}(G)^{H} \cap \mathcal{A}_{p}(C E)\right)_{\geq E}\right)$. The space $B\left(\left(\mathcal{A}_{p}(G)^{H} \cap \mathcal{A}_{p}(C E)\right)_{\geq E}\right)$ is contractible because $\mathcal{A}_{p}(G)_{\geq E}^{H}$ has the final object $E$. Now we can apply 1.4(i).

If $G$ is finite and a normal subgroup $H$ of $G$ contains a non-trivial $p$ subgroup then it was proved in $[\mathrm{Dw}]$ that the space $B \mathcal{A}_{p}(G) / H$ is $F_{p}$-acyclic. In 1.6 we will prove that this space is contractible.

Let $\mathcal{K}_{p}(G)$ denote the set of all subgroups $H$ of $G$ satisfying the condition that, for every maximal $p$-toral subgroup $P$ in $G, H \cap Z(P)$ contains a nontrivial $p$-subgroup. If $H \in \mathcal{K}_{p}(G)$ and $H \subseteq H^{\prime}$, then $H^{\prime} \in \mathcal{K}_{p}(G)$. If $H$ is a normal subgroup of $G$ which, for every maximal $p$-toral subgroup $P$ of $G$, contains a non-trivial normal $p$-toral subgroup $P^{\prime}$ of $P$, then $H \in \mathcal{K}_{p}(G)$. Indeed, $H \cap Z P$ contains $P^{\prime P}$, hence it contains a non-trivial $p$-group. If $G$ is finite and a normal subgroup $H$ of $G$ contains a non-trivial $p$-subgroup, then $H$ belongs to $\mathcal{K}_{p}(G)$. It was proved in [Dw] that in this case $H \cap P$ is a normal subgroup of $P$ and a Sylow $p$-subgroup of $H$ so $H \cap Z(P)$ contains a non-trivial $p$-subgroup. The following result is a generalization of 1.5 .

1.6. Proposition. Let $G$ be compact Lie group with a non-trivial $p$ subgroup. If $H \in \mathcal{K}_{p}(G)$ then the space $B \mathcal{A}_{p}(G) / H$ is contractible.

Proof. The result is a consequence of 1.4(i). It follows from the definition that $\mathcal{N}_{p}(H) \subseteq \mathcal{N}_{p}(G)$, hence, as in the proof of 1.5, for every $H_{0} \in \mathcal{N}_{p}(H)$, $B \mathcal{A}_{p}(G)^{H_{0}}$ is contractible. If $x \in B \mathcal{A}_{p}(G)$, then $H_{x}$ contains a non-trivial p-subgroup. Indeed, let $G_{x}=N E_{0} \cap \ldots \cap N E_{k}$, where $E_{i} \in \mathcal{A}_{p}(G)$ and $E_{0}<\ldots<E_{k}$. Let $P$ be a maximal $p$-toral subgroup of $G$ such that $E_{k} \subseteq P$. It follows from the definitions that $H \cap Z P \subseteq H \cap N E_{0} \cap \ldots \cap N E_{k}=H_{x}$. The assumption that $H \in \mathcal{K}_{p}(G)$ now implies that $H_{x}$ contains a non-trivial $p$-subgroup.

2. Homotopy decompositions over $(\operatorname{sd} W) / G$. Let $\mathcal{C}$ be a topological category. For any two functors $Y: \mathcal{C} \rightarrow$ Top and $Y^{\prime}: \mathcal{C}^{\text {op }} \rightarrow$ Top, the topological space $Y^{\prime} \times_{\mathcal{C}} Y$ is the coequalizer of the two natural maps

$$
p_{0}, p_{1}: \coprod_{\alpha: c \rightarrow c^{\prime}} Y(c) \times Y^{\prime}\left(c^{\prime}\right) \rightarrow \coprod_{c \in C} Y(c) \times Y^{\prime}(c)
$$

induced by the maps

$$
p_{0}(\alpha)\left(y, y^{\prime}\right)=\left(Y(\alpha) y, y^{\prime}\right), \quad p_{1}(\alpha)\left(y, y^{\prime}\right)=\left(y, Y^{\prime}(\alpha) y^{\prime}\right) .
$$

In particular $\operatorname{hocolim}_{\mathcal{C}} Y=B(-\downarrow \mathcal{C}) \times_{\mathcal{C}} Y$, where $c \downarrow \mathcal{C}$ is the "under" category of the morphisms $c \rightarrow c^{\prime}$ of $\mathcal{C}$. 
If $G_{1}$ and $G_{2}$ are groups and $Y: \mathcal{C} \rightarrow G_{1}$-Top, $Y^{\prime}: \mathcal{C}^{\text {op }} \rightarrow G_{2}$-Top, then $G_{1} \times G_{2}$ acts in a natural way on $Y^{\prime} \times_{\mathcal{C}} Y$. If $G_{1}=e$ then we obtain a $G_{2}$-action.

Let $G$ be a Lie group and let $X$ be a $G$-CW-complex. Let $\mathcal{S}(G, X)$ denote the set of isotropy groups of $X$ and $\mathcal{S}_{0}(G, X)=\mathcal{S}(G, X) \cup\{G\}$. The full subcategory of $\mathcal{O}_{G}$ whose objects are the orbit spaces $G / H$, where $H \in \mathcal{S}(G, X)$, is denoted by $\mathcal{O}(G, X)$. The $G$-map spaces will be denoted by $\operatorname{Map}_{G}(-,-)$.

Let $F_{1}, F_{2}: G$-CW $\rightarrow G$-CW be functors such that

$$
F_{i}(X)=\operatorname{Map}_{G}(-, X) \times_{\mathcal{O}_{G}} F_{i}, \quad F_{i}(f)=\operatorname{Map}_{G}(-, f) \times_{\mathcal{O}_{G}} F_{i}
$$

whenever $f: X \rightarrow X^{\prime}$. In the formulas above the restriction of $F_{i}$ to the subcategory $\mathcal{O}_{G}$ of $G$-CW is denoted by the same letter. We will need the following fact.

2.1. Proposition. Let $\tau: F_{1} \rightarrow F_{2}$ be a natural transformation of functors induced by its restriction to $\mathcal{O}_{G}$. If , for every $G / H \in \mathcal{O}(G, X), \tau(G / H)$ is a G-homotopy equivalence, then so is $\tau(X): F_{1}(X) \rightarrow F_{2}(X)$.

Proof. Since the $\mathcal{O}_{G}$-orbits of the functor $\operatorname{Map}_{G}(-, X)$ have the form $\operatorname{Map}_{G}\left(-, G / G_{x}\right)$, where $x \in X$, the restriction of $\operatorname{Map}_{G}(-, X)$ to $\mathcal{O}(G, X)$ is a free functor in the sense of [DF1] and

$$
F_{i}(X)=\operatorname{Map}_{G}(-, X) \times_{\mathcal{O}(G, X)} F_{i} .
$$

This can be proved by induction on the dimension of $X$. Assume that the $n$-skeleton of $X$, denoted by $X_{n}$, is equal to the pushout

$$
D^{n} \times T_{n} \leftarrow S^{n-1} \times T_{n} \rightarrow X_{n-1}
$$

where $T_{n}$ is a disjoint union of $G$-orbits from $\mathcal{O}(G, X)$ and the left arrow is the cofibration induced by the natural inclusion $S^{n-1} \rightarrow D^{n}$. Then $F_{i}\left(X_{n}\right)$ is equal to the homotopy pushout

$$
D^{n} \times F_{i}\left(T_{n}\right) \leftarrow S^{n} \times F_{i}\left(T_{n}\right) \rightarrow F_{i}\left(X_{n-1}\right) .
$$

This implies that, if $\tau\left(X_{n-1}\right)$ is a homotopy equivalence then so is $\tau\left(X_{n}\right)$. Now one can use the fact that $\tau(X)=\operatorname{hocolim}_{n \in \mathbb{N}} \tau\left(X_{n}\right)$.

2.2. Examples. (i) Let $K$ be a $G$-CW-complex. Let $F=\left(H(-), H^{\prime}(-)\right)$ : $\mathcal{C} \rightarrow \mathcal{C}_{G}$ be a functor such that, for every isotropy group $G^{\prime}$ of $X$, the map

$$
\operatorname{hocolim}_{c \in \mathcal{C}} K \times_{H(c)}\left(G / G^{\prime}\right)^{H^{\prime}(c)} \rightarrow K / G^{\prime}
$$

is a homotopy equivalence. Then so is the map

$$
\operatorname{hocolim}_{c \in \mathcal{C}} K \times_{H(c)} X^{H^{\prime}(c)} \rightarrow K \times_{G} X \text {. }
$$

(ii) Let $f: K_{1} \rightarrow K_{2}$ be a cellular map of $G$-CW-complexes. If, for every isotropy group $H$ of $X, f / H: K_{1} / H \rightarrow K_{2} / H$ is a homotopy equivalence, then so is $f \times_{G} X: K_{1} \times_{G} X \rightarrow K_{2} \times_{G} X$. 
(iii) Let $V$ be a $G$-subposet of $\mathcal{S}(G)$. Using the fact that for every $G^{\prime} \in V$, $\operatorname{hocolim}_{G / H \in \mathcal{O}_{V}}\left(G / G^{\prime}\right)^{H}=B\left(G / G^{\prime} \downarrow \mathcal{O}_{V}\right) \simeq *$,

we obtain the decomposition described in 0.0 (ii).

(iv) Let $F=\left(H(-), H^{\prime}(-)\right): \mathcal{C} \rightarrow \mathcal{C}_{G}$ be a functor such that, for every isotropy group $G^{\prime}$ of $X$, the map

$$
\operatorname{hocolim}_{c \in \mathcal{C}} G \times_{H(c)}\left(G / G^{\prime}\right)^{H^{\prime}(c)} \rightarrow G / G^{\prime}
$$

is a $G$-homotopy equivalence. Then so is the map

$$
\operatorname{hocolim}_{c \in \mathcal{C}} G \times_{H(c)} X^{H^{\prime}(c)} \rightarrow X .
$$

In this section we will assume that $W$ is a topological $G$-subposet of $\mathcal{S}(G)$ and that all elements of $W$ are finite subgroups of $G$. This implies that the orbit spaces $d_{n} W / G$ are discrete and that $W$ satisfies the condition that $w \leq g w$, where $g \in G$, implies that $w=g w$.

Let $H$ be a closed subgroup of $G$. We will use the notation

$$
W_{H}=\left\{H^{\prime} \in W: H^{\prime} \subseteq H\right\} .
$$

If $H$ is a compact Lie group then the topology on $W_{H}$ induced from $W$ is equal to the topology induced from $\mathcal{S}(H)$. This follows from the fact that $(G / H)^{H^{\prime}} / N H^{\prime}$ is discrete (cf. the proof of II.5.7 in [Br2]).

2.3. Proposition. Let $X$ be a $G$-CW-complex such that all its isotropy groups are compact.

(i) If, for every $x \in X$, the map $K \times_{G_{x}} B\left(W_{G_{x}}\right) \rightarrow K / G_{x}$ is a homotopy equivalence, then there is a homotopy decomposition

$\operatorname{hocolim}_{\left[\left(H_{0}, \ldots, H_{n}\right)\right] \in \operatorname{sd} W / G} K \times_{N H_{0} \cap \ldots \cap N H_{n}} X^{H_{n}} \simeq K \times_{G} X$.

(ii) If, for every $x \in X$, the map $G \times \times_{G_{x}} B\left(W_{G_{x}}\right) \rightarrow G / G_{x}$ is a $G$ homotopy equivalence, then there is a $G$-homotopy decomposition

$$
\operatorname{hocolim}_{\left[\left(H_{0}, \ldots, H_{n}\right)\right] \in \operatorname{sd} W / G} G \times_{N H_{0} \cap \ldots \cap N H_{n}} X^{H_{n}} \simeq X .
$$

Proof. Let

$$
F_{K}^{\prime}(X)=\operatorname{hocolim}_{\left[\left(H_{0}, \ldots, H_{n}\right)\right] \in \operatorname{sd} W / G} K \times_{N H_{0} \cap \ldots \cap N H_{n}} X^{H_{n}} .
$$

It follows from the definitions that $F_{K}^{\prime}(X)=K \times_{G} F_{G}^{\prime}(X)$.

If $X=*=G / G$, then there is a $G$-homotopy equivalence

$$
F_{G}^{\prime}(*)=\operatorname{hocolim}_{\left[\left(H_{0}, \ldots, H_{n}\right)\right] \in \operatorname{sd} W / G} G /\left(N H_{0} \cap \ldots \cap N H_{n}\right) \simeq B W .
$$

Indeed, $F_{G}^{\prime}(*)$ is the classifying space of the category $W[G]$ whose objects are the pairs $([w],.[g])$, where $[w.] \in \operatorname{sd} W / G,[g] \in G /\left(N H_{0} \cap \ldots \cap\right.$ $\left.N H_{n}\right), w .=\left(H_{0}, \ldots, H_{n}\right)$. The category $W[G]$ is a topological poset with an action of $G$ defined by the action of $G$ on $G / G_{w}$. and there is an equivariant isomorphism of topological $G$-posets $F: W[G] \rightarrow \operatorname{sd} W$ such that $F([w],.[g])=g w$.. Hence we have equivariant homotopy equivalences 
$F_{G}^{\prime}(*) \simeq B W[G] \simeq B$ sd $W$. Let $\mathcal{N}$ be the category whose objects are finite posets $[n]=\{0 \leq 1 \leq \ldots \leq n\}$ and whose morphisms are the injective poset maps. Let $F_{W}: \mathcal{N} \rightarrow G$-Top be the functor such that $F_{W}([n])=\operatorname{sd}_{n} W$ consists of all injective poset maps $[n] \rightarrow W$. Let $\Delta_{n}$ be the standard $n$ dimensional simplex. Then $\Delta_{(-)}$is a free functor on the category $\mathcal{N}$. This implies that there are equivariant homotopy equivalences

$$
B \operatorname{sd} W \simeq \operatorname{hocolim}_{\mathcal{N}} F_{W} \simeq \Delta_{(-)} \times_{\mathcal{N}} \operatorname{sd}_{(-)} W \simeq B W .
$$

There is a natural $G$-CW-complex structure on $B W$ such that the poset $\operatorname{sd} W / G=(\operatorname{sd} W) / G$ is equal to the poset of the $G$-cells of $B W$. For $K=*$ we obtain homotopy equivalences

$$
B((\operatorname{sd} W) / G)=F_{*}^{\prime}(*)=F_{G}^{\prime}(*) / G \simeq B(\operatorname{sd} W) / G \simeq(B W) / G .
$$

The inclusions $X^{H_{n}} \rightarrow X$ induce a map $p_{K}(X): F_{K}^{\prime}(X) \rightarrow K \times_{G} X$. The $\operatorname{map} p_{G}(X)$ is a $G$-map and $p_{K}(X)=K \times_{G} p_{G}(X)$. Let $\pi_{X}: F_{G}^{\prime}(X) \rightarrow$ $F_{G}^{\prime}(*) \simeq B W$ be the natural $G$-projection. To obtain the result it is sufficient to prove that, for every $x \in X$, the map $p_{K}\left(G / G_{x}\right)$ is a homotopy equivalence. This follows from the fact that, for every closed subgroup $H$ of $G, \pi_{X}$ induces an $H$-homotopy equivalence $p_{G}^{-1}(G / H)(H) \rightarrow B W_{H}$. Indeed, consider the natural projection $f_{w}$ : $G \times_{N H_{0} \cap \ldots \cap N H_{n}}(G / H)^{H_{n}} \rightarrow G / H$. Then $G \times_{N H_{0} \cap \ldots \cap N H_{n}}(G / H)^{H_{n}}=G \times_{H} f_{w}^{-1}(H)$. Let

$$
\begin{aligned}
Y(w ., H) & =\left\{g \in G: g H_{n} g^{-1} \subseteq H\right\} /\left(N H_{0} \cap \ldots \cap N H_{n}\right) \\
& \subseteq G /\left(N H_{0} \cap \ldots \cap N H_{n}\right) .
\end{aligned}
$$

Then there is an $H$-isomorphism $\mu: Y(w ., H) \rightarrow f_{w}^{-1}(H)$ such that $\mu([g])=$ $\left[g, g^{-1} H\right]$. The space

$$
p_{G}^{-1}(G / H)(H) \simeq \operatorname{hocolim}_{[w .] \in \operatorname{sd} W / G} Y(w ., H)
$$

is the classifying space of the category $W[H]$ whose objects are the pairs ([w.], $[g])$, where $[w.] \in \operatorname{sd} W / G,[g] \in Y(w ., H) . W[H]$ is a topological subposet of $W[G]$ and the restriction of $F_{W}$ gives us an $H$-poset isomorphism $W[H] \rightarrow \operatorname{sd} W_{H}$. Now we can use the $H$-homotopy equivalence $B \operatorname{sd} W_{H} \simeq$ $B W_{H}$ to conclude that $p_{K}(G / H)$ is homotopy equivalent to the projection $K \times_{H} B W_{H} \rightarrow K / H$ (which implies (i)) and that $p_{G}(G / H)$ is $G$-homotopy equivalent to the projection $G \times_{H} B W_{H} \rightarrow G / H$ (which implies (ii)).

The following result is an immediate consequence of 2.3 .

2.4. Corollary. Let $X$ be a $G$-CW-complex such that all its isotropy groups are compact. Let $W$ be a $G$-poset of finite subgroups of $G$ such that the space $B \operatorname{sd} W / G$ is contractible. Suppose that $\mathcal{A}$ is thick and satisfies the condition A1. 
(i) Suppose that, for every $x \in X$, the map $K \times_{G_{x}} B\left(W_{G_{x}}\right) \rightarrow K / G_{x}$ is a homotopy equivalence and that, for every $\left(H_{0}, \ldots, H_{n}\right) \in \operatorname{sd} W$, we have $K \times_{N H_{0} \cap \ldots \cap N H_{n}} X^{H_{n}} \in \mathcal{A}$. Then $K \times_{G} X \in \mathcal{A}$.

(ii) Suppose that, for every $x \in X$, the space

$$
B\left(W_{G_{x}}\right) / G_{x}=B \operatorname{sd} W_{G_{x}} / G_{x}
$$

is contractible and that $X^{H} \in \mathcal{A}$ whenever

$$
\begin{aligned}
H \in\left\{N H_{0} \cap \ldots \cap N H_{n} \cap G^{\prime}:\left(H_{0}, \ldots, H_{n}\right)\right. & \in \operatorname{sd} W, \\
G^{\prime} & \left.\in \mathcal{S}_{0}(G, X), H_{n} \subseteq G^{\prime}\right\} .
\end{aligned}
$$

Then $X / G \in \mathcal{A}$.

2.5. Examples. (i) Let $X$ be a $G$-CW-complex such that all its isotropy groups are finite. Then there exists a $G$-homotopy decomposition

$$
\operatorname{hocolim}_{\left[\left(H_{0}, \ldots, H_{n}\right)\right] \in \operatorname{sd} \mathcal{S}(G, X) / G} G \times_{N H_{0} \cap \ldots \cap N H_{n}} X^{H_{n}} \simeq X
$$

because, for every $x \in X$, the space $B \mathcal{S}(G, X)_{G_{x}}$ is $G_{x}$-contractible.

(ii) Suppose that, for every $x \in X, y \in K, G_{x} \in \mathcal{S}_{\mathrm{c}}(G)$ and $G_{x} \cap G_{y} \in$ $\mathcal{K}_{p}\left(G_{x}\right)$. Then there is a homotopy equivalence

$\operatorname{hocolim}_{\left[\left(E_{0}, \ldots, E_{n}\right)\right] \in \operatorname{sd} \mathcal{A}_{p}(G) / G} K \times_{N E_{0} \cap \ldots \cap N E_{n}} X^{E_{n}} \simeq K \times_{G} X$.

This is a consequence of 2.3, 2.2(ii) and 1.6. In particular, for $K=*$ we obtain 0.1 .

(iii) Let $G$ be compact Lie group with a non-trivial $p$-subgroup. Let $\mathcal{P}$ be the poset of all non-trivial finite $p$-subgroups of $G$. Then the space $(B \mathcal{P}) / G$ is contractible. This follows from (ii) and from the fact that, for every $\left(E_{0}, \ldots, E_{n}\right) \in \operatorname{sd} \mathcal{A}_{p}(G)$, the space $B(\mathcal{P})^{H}$ is contractible whenever $E_{n} \leq H \leq N E_{0} \cap \ldots \cap N E_{n}$ because $P^{\prime} E_{n} \in \mathcal{P}^{H}$ if $P^{\prime} \in \mathcal{P}^{H}$.

(iv) Let $X$ be a $G$-CW-complex such that all its isotropy groups are compact and contain a non-trivial normal $p$-subgroup. Then there exists a $G$-homotopy decomposition

$$
\operatorname{hocolim}_{\left[\left(E_{0}, \ldots, E_{n}\right)\right] \in \operatorname{sd} \mathcal{A}_{p}(G) / G} G \times_{N E_{0} \cap \ldots \cap N E_{n}} X^{E_{n}} \simeq X
$$

because, for every $x \in X$, the space $B \mathcal{A}_{p}\left(G_{x}\right)$ is $G_{x}$ contractible. This follows from the fact that the poset $\mathcal{A}_{p}\left(G_{x}\right)^{G_{x}}$ is non-empty (cf. the proof of 1.5), and that, for every isotropy group $H$ of $B \mathcal{A}_{p}\left(G_{x}\right)$, the map $B \mathcal{A}_{p}\left(G_{x}\right)^{H} \rightarrow *$ is a homotopy equivalence because all isotropy groups of $B \mathcal{A}_{p}\left(G_{x}\right)$ contain non-trivial normal $p$-subgroups.

One can prove this fact using similar methods to those in 1.5. Let $E$ be a non-trivial, normal, elementary abelian $p$-subgroup of $G_{x}$. Let $W$ be the $G$-poset of all subgroups of $G_{x}$ of the form $E^{\prime} E^{\prime \prime}$ where $E^{\prime} \in \mathcal{A}_{p}\left(G_{x}\right)$ and $E^{\prime \prime}$ is a subgroup of $E$. Then $B W$ is $G_{x}$-contractible. 
Let $G$ be a finite group. If $\mathcal{P}$ is a concave $G$-poset of $p$-subgroups of $G$, then $\mathcal{P}^{o}$ is the $G$-subposet of $\mathcal{P}$ such that $P \in \mathcal{P}^{o}$ if and only if $P \in \mathcal{P}$ and $\Phi(P) \notin \mathcal{P}$. Here $\Phi(P)$ denotes the Frattini subgroup of $P$. If $\mathcal{P}=\mathcal{T}_{p}(G)$, then $\mathcal{P}^{o}=\mathcal{A}_{p}(G)$.

2.6. Proposition. Let $G$ be a finite group. Let $\mathcal{P}^{\prime}$ be a concave $G$-poset of p-subgroups of $G$. Let $X$ be a $G$-CW-complex such that all Sylow $p$ subgroups of its isotropy groups are in $\mathcal{P}^{\prime}$. Suppose that $\mathcal{P}$ is a G-poset of p-subgroups of $G$ such that $\mathcal{P}^{\prime o} \subseteq \mathcal{P} \subseteq \mathcal{P}^{\prime}$. Then there is a homotopy equivalence

$$
\operatorname{hocolim}_{\left[\left(P_{0}, \ldots, P_{n}\right)\right] \in \operatorname{sd} \mathcal{P} / G} X^{P_{n}} /\left(N P_{0} \cap \ldots \cap N P_{n}\right) \simeq X / G .
$$

Proof. The space $B\left(\mathcal{P}^{\prime}\right) / G$ is contractible. (This is a generalization of Corollary 2.6.1 of [We], which states that $B\left(\mathcal{P}^{\prime}\right) / G$ is $F_{p^{-a c y c l i c}}$.) Indeed, if $x \in B\left(\mathcal{P}^{\prime}\right)$, then $G_{x}=N P_{0} \cap \ldots \cap N P_{k}$, where $P_{i} \in \mathcal{P}^{\prime}$ and $P_{0}<$ $\ldots<P_{k}$, so Sylow $p$-subgroups of $G_{x}$ are in $\mathcal{P}^{\prime}$. It is proved in [We] (2.1.2) that, for every $H \in \mathcal{N}_{\mathcal{P}^{\prime}}$, the space $B\left(\mathcal{P}^{\prime}\right)^{H}$ is contractible. Thus we can apply 1.2 (ii) to the class $\mathcal{C}$. Proposition 1.7 of [TW] implies that the $H$-map $B\left(\mathcal{P}_{H}\right) \rightarrow B\left(\mathcal{P}_{H}^{\prime}\right)$, induced by the inclusion of $H$-posets of subgroups, is an $H$-homotopy equivalence. The proof of this fact is similar to the proof of 2.1(i) of [TW]. Hence $B\left(\mathcal{P}_{H}\right) / H \simeq B\left(\mathcal{P}_{H}^{\prime}\right) / H$ and the space $B\left(\mathcal{P}_{H}\right) / H$ is contractible. Now we can use 2.1.

The following result is an immediate consequence of 2.6. It is stronger than 1.2 .

2.7. Corollary. Let $G$ be a finite group. Let $\mathcal{P}$ and $X$ satisfy the assumptions of 2.6. Suppose that $\mathcal{A}$ is thick and satisfies the condition A1 and that one of the following conditions holds:

(i) $X^{P_{n}} /\left(N P_{0} \cap \ldots \cap N P_{n}\right) \in \mathcal{A}$ whenever $\left(P_{0}, \ldots, P_{n}\right) \in \operatorname{sd} \mathcal{P}$,

(ii) $X^{H} \in \mathcal{A}$ whenever

$$
\begin{aligned}
H \in\left\{N P_{0} \cap \ldots \cap N P_{n} \cap G^{\prime}:\left(P_{0}, \ldots, P_{n}\right)\right. & \in \operatorname{sd} \mathcal{P}, \\
& \left.G^{\prime} \in \mathcal{S}_{0}(G, X), P_{n} \subseteq G^{\prime}\right\} .
\end{aligned}
$$

Then $X / G \in \mathcal{A}$.

2.8. Corollary. Let $G$ be a finite group. Let $\mathcal{P}$ be a $G$-poset of $p$ subgroups of $G$ such that $\mathcal{A}_{p}(G) \subseteq \mathcal{P}$. If , for every $x \in X$ and $y \in K, G_{x}$ contains a non-trivial p-subgroup and $G_{x} \cap G_{y} \in \mathcal{K}_{p}\left(G_{x}\right)$, then there is a homotopy equivalence

$$
\operatorname{hocolim}_{\left[\left(P_{0}, \ldots, P_{n}\right)\right] \in \operatorname{sd} \mathcal{P} / G} K \times_{N P_{0} \cap \ldots \cap N P_{n}} X^{P_{n}} \simeq K \times_{G} X .
$$

Proof. This result is a consequence of 2.5 (ii). Let $P$ be a non-trivial $p$-subgroup of $G$. It follows from [TW], 1.7 and 2.1, that there is an $H$ - 
homotopy equivalence $B\left(\mathcal{A}_{p}(G)_{H}\right) \rightarrow B\left(\mathcal{P}_{H}\right)$ whenever $H$ is a subgroup of $G$ and contains a non-trivial $p$-subgroup. Now we can use 2.1 and 2.3 .

3. Categories associated to $G$-posets. Let $K$ be a $G$-CW-complex. Every equivariant cellular map $f: X_{1} \rightarrow X_{2}$ of $G$-CW-complexes induces maps $f\left(H, H^{\prime}\right): K \times_{H} X_{1}^{H^{\prime}} \rightarrow K \times_{H} X_{2}^{H^{\prime}}$ where $\left(H, H^{\prime}\right) \in \mathcal{W}(G)$, i.e. $H, H^{\prime} \in \mathcal{S}(G)$ and $H \subseteq N H^{\prime}$.

For every functor $F=\left(H(-), H^{\prime}(-)\right): \mathcal{C} \rightarrow \mathcal{C}_{G}$ we have maps

$$
\begin{gathered}
\phi_{i}: \operatorname{hocolim}_{c \in \mathcal{C}} K \times_{H(c)} X_{i}^{H^{\prime}(c)} \rightarrow K \times_{G} X_{i}, \\
f_{F}: \operatorname{hocolim}_{c \in \mathcal{C}} K \times_{H(c)} X_{1}^{H^{\prime}(c)} \rightarrow \operatorname{hocolim}_{c \in \mathcal{C}} K \times_{H(c)} X_{2}^{H^{\prime}(c)}
\end{gathered}
$$

such that $f_{F}=\operatorname{hocolim}_{c \in \mathcal{C}} f\left(H(c), H^{\prime}(c)\right)$ and $f(G, e) \phi_{1}=\phi_{2} f_{F}$. It follows from general homotopy colimit theory that, if $f\left(H(c), H^{\prime}(c)\right)$ are homotopy equivalences for all $c \in \mathcal{C}$, then the map $f(G, e): K \times_{G} X_{1} \rightarrow K \times_{G} X_{2}$ is a homotopy equivalence. This motivates the following definition.

3.0. Definition. Let $\mathcal{S}$ be a $G$-poset of closed subgroups of $G$. A $G$ subposet $W$ of $\mathcal{W}(G)$ is $(\mathcal{S}, K)$-essential if, for every equivariant cellular map $f: X \rightarrow Y$ of $G$-CW-complexes with all isotropy groups in $\mathcal{S}$, the condition that $K \times_{H} X^{H^{\prime}} \rightarrow K \times_{H} Y^{H^{\prime}}$ is a homotopy equivalence for every $\left(H, H^{\prime}\right) \in W$ implies that $K \times_{G} X \rightarrow K \times_{G} Y$ is a homotopy equivalence.

In particular, if $W$ is $\left(\mathcal{S}_{0}(G, X), K\right)$-essential and $K \times_{H} X^{H^{\prime}} \rightarrow K / H$ is a homotopy equivalence whenever $\left(H, H^{\prime}\right) \in W$, then $K \times_{G} X \rightarrow K / G$ is a homotopy equivalence.

The results of previous sections enable us to exhibit many non-trivial examples of essential posets. Our main tool will be the following consequence of $2.2(\mathrm{i})$.

\subsection{Proposition. Suppose that}

$$
F=\left(H(-), H^{\prime}(-)\right): \mathcal{C} \rightarrow \mathcal{C}_{G}\left(W, d_{\mathcal{W}(G)}\right)
$$

is a functor such that for every $G^{\prime} \in \mathcal{S}$, the map

$$
\operatorname{hocolim}_{c \in \mathcal{C}} K \times_{H(c)}\left(G / G^{\prime}\right)^{H^{\prime}(c)} \rightarrow K / G^{\prime}
$$

is a homotopy equivalence. Then the poset $W$ is $(\mathcal{S}, K)$-essential.

3.2. ExAmples. (i) Let $\mathcal{P}$ be a concave $G$-subposet of $p$-toral subgroups of $G$ such that all maximal $p$-toral subgroups of elements of $\mathcal{S}$ are in $\mathcal{P}$. Then it follows from 1.1 that the poset $\mathcal{W}_{\mathcal{P}}=\{(H, P): P \subseteq H \subseteq N P$, $\left.P \in \mathcal{P}, H \in \mathcal{S}_{\mathrm{c}}^{\prime}(G)\right\}$ is $(\mathcal{S}, *)$-essential.

(ii) The poset $\mathcal{W}_{\mathcal{A}_{p}(G)}=\left\{(H, E): E \subseteq H \subseteq N E, E \in \mathcal{S}, H \in \mathcal{S}_{\mathrm{c}}^{\prime}(G)\right\}$ is $\left(\mathcal{S}_{\mathrm{c}}(G), *\right)$-essential. Let $\mathcal{S}_{K}(G)$ be the poset of all compact subgroups $H$ of $G$ with non-trivial $p$-subgroups and such that $H \cap G_{k} \in \mathcal{K}_{p}(H)$ for 
every $k \in K$. Then the poset $\mathcal{W}_{\mathcal{A}_{p}(G)}$ is also $\left(\mathcal{S}_{K}(G), K\right)$-essential. This is a consequence of 2.5 (ii).

(iii) Let $f: X \rightarrow Y$ be an equivariant cellular map of $G$-CW-complexes such that, for every compact subgroup $H$ of $G$ with a non-trivial normal $p$-toral subgroup, the map $f^{H}: X^{H} \rightarrow Y^{H}$ is a homotopy equivalence. This implies that, for every $\left(H, H^{\prime}\right) \in \mathcal{W}_{\mathcal{A}_{p}(G)}$, the map $f^{H^{\prime}}$ is an $H$-homotopy equivalence so the map $K \times_{H} X^{H^{\prime}} \rightarrow K \times_{H} Y^{H^{\prime}}$ is a homotopy equivalence. If all isotropy groups of points of $X$ and $Y$ are in $\mathcal{S}_{K}(G)$, then, by (ii), the map $F_{K}(f): K \times_{G} X \rightarrow K \times_{G} Y$ is also a homotopy equivalence. In the case when $K=*$ we obtain $0.3(\mathrm{i})$.

Now we describe a construction of topological categories $\mathcal{C}$ associated to topological $G$-posets and some examples of functors $\mathcal{C} \rightarrow \mathcal{C}_{G}$ defined on such categories. We show that the known homotopy and homology decompositions can be obtained using this construction.

Let $W$ be a topological $G$-poset such that $W / G$ is a discrete topological space. Let $d: W \rightarrow \mathcal{S}(G)$ be a $G$-poset map such that, for every $w \in W, d w$ is a subgroup of $G_{w}$. It follows that $d w$ is a closed normal subgroup of $G_{w}$. The $G$-poset maps with the above property will be called admissible maps. Let $\mathcal{C}_{G}(W, d)$ be the topological category whose objects are the elements of $W$ and whose morphism spaces are defined by

$$
\operatorname{Mor}_{\mathcal{C}_{G}(W, d)}\left(w, w^{\prime}\right)=\left\{g \in G: w \leq g w^{\prime}\right\} / d w^{\prime} \subseteq G / d w^{\prime} .
$$

The composition of $[g]: w \rightarrow w^{\prime}$ and $\left[g^{\prime}\right]: w^{\prime} \rightarrow w^{\prime \prime}$ is $\left[g g^{\prime}\right]: w \rightarrow w^{\prime \prime}$. The categories $\mathcal{C}_{G}(W, d)$, for discrete groups $G$, are studied in [S1-3], [JS].

3.3. Examples. (i) Let $W(G)$ denote the $G$-subposet of $\mathcal{S}(G) \times W$ whose elements are all pairs $(H, w)$ where $w \in W$ and $H \subseteq G_{w}$. Let $d_{W(G)}$ be the admissible map $W(G) \rightarrow \mathcal{S}(G)$ such that $d_{W(G)}(H, w)=H$. Let $\mathcal{C}_{G}\left(W(G), d_{W(G)}\right)=\mathcal{C}_{G}(W)$. It follows from the definitions that $\mathcal{C}_{G}(*)=$ $\mathcal{O}_{G}$. If $p_{W}: W(G) / G \rightarrow \mathcal{S}(G) / G$ is the map induced by the natural projection, then, for every closed subgroup $H$ of $G, p_{W}^{-1}([H])=W^{H} / N H$. (In the notation of $[\mathrm{T}], \mathcal{C}_{G}(W)=\int_{H \in \mathcal{O}_{G}} W^{H}$.) The space $W(G) / G$ is discrete if, for every $H \in \mathcal{S}(G), W^{H} / N H$ is discrete. Hence if, for every $w \in W$, $\left(G / G_{w}\right)^{H} / N H$ is discrete then $W(G) / G$ is a discrete space. This is, in particular, the case when, for every $w \in W, G_{w}$ is compact (cf. II.5.7 of [Br2]).

(ii) Let $d: W \rightarrow \mathcal{S}(G)$ be an arbitrary admissible function. Then there exists an inclusion $j_{d}: \mathcal{C}_{G}(W, d) \rightarrow \mathcal{C}_{G}(W)$ such that $j_{d}(w)=(d w, w)$ and the image of $j_{d}$ is a full subcategory of $\mathcal{C}_{G}(W)$.

(iii) For $W=\mathcal{S}(G)^{\mathrm{op}}, W(G)=\mathcal{W}(G)$ and $\mathcal{C}_{G}(W)=\mathcal{C}_{G}$. Let $V$ be a $G$-set of subgroups of $G$. Denote by $\mathcal{W}(V)$ the $G$-subposet of $\mathcal{W}(G)$ such that $\left(H, H^{\prime}\right) \in \mathcal{W}(V)$ if and only if $H, H^{\prime} \in V$ and $H \subseteq H^{\prime}$. The full subcategory of $\mathcal{C}_{G}$ whose object set is $\mathcal{W}(V)$ will be denoted by $\mathcal{C}(V)$. If 
$p: \mathcal{W}(V) / G \rightarrow \mathcal{S}(G) / G$ is induced by the natural projection, then, for every closed subgroup $H$ of $G, p^{-1}([H])=V(\geq H) / N H$, where $V(\geq H)$ is the set of all elements of $V$ which contain $H$. (That is, $\mathcal{C}(V)=\int_{H \in \mathcal{O}_{V}} V(\geq H)$.) Hence the space $\mathcal{W}(V)$ is discrete if, for every $H, H^{\prime} \in V, H \subseteq H^{\prime}$ implies that $\left(N H^{\prime} \backslash\left(G / H^{\prime}\right)^{H}\right) / N H$ is discrete. In particular, if $V$ is a $G$-poset of compact subgroups of $G$, then $\mathcal{W}(V) / G$ is discrete (II.5.7 of [Br2]).

(iv) Let $U$ be a $G$-space and let $W$ be a $G$-poset of non-empty finite subsets of $U$. There exists an admissible function $d_{U}$ such that, for every $w \in W, d_{U} w=\bigcap_{u \in w} G_{u}$.

There exists a functor $O_{d}: \mathcal{C}_{G}(W, d) \rightarrow \mathcal{O}_{G}$ such that $O_{d}(w)=G / d w$ for every $w \in W$, and $O_{d}([g])\left(g^{\prime} d w\right)=g^{\prime} g d w^{\prime}$ for every morphism $[g]: w \rightarrow w^{\prime}$ of $\mathcal{C}_{G}(W, d)$. We will use the notation

$$
E_{G}(W, d)=\operatorname{hocolim}_{w \in \mathcal{C}_{G}(W, d)} G / d w .
$$

Let $d^{\prime}: W^{\text {op }} \rightarrow \mathcal{S}(G)$ be a $G$-poset map. Then, for every $w \in W$, $d w \subseteq G_{w} \subseteq N d^{\prime} w$. Hence there exists a functor $\left(d, d^{\prime}\right): \mathcal{C}_{G}(W, d) \rightarrow \mathcal{C}_{G}$ such that $\left(d, d^{\prime}\right)(w)=\left(d w, d^{\prime} w\right)$.

Let $G^{\prime}$ be a subgroup of $G$. We will use the notation

$$
W_{d^{\prime}, G^{\prime}}=\left\{w \in W: d^{\prime} w \subseteq G^{\prime}\right\} .
$$

$W_{d^{\prime}, G^{\prime}}$ will be considered as a $G^{\prime}$-poset. The admissible function $d_{G^{\prime}}$ : $W_{d^{\prime}, G^{\prime}} \rightarrow \mathcal{S}\left(G^{\prime}\right)$ will be defined in such a way that, for every $w \in W_{d^{\prime}, G^{\prime}}$, $d_{G^{\prime}} w=G^{\prime} \cap d w$.

3.4. Lemma. Let $G^{\prime}$ be a closed subgroup of $G$ such that $W_{d^{\prime}, G^{\prime}} / G^{\prime}$ is a discrete space. Then there exists a G-homotopy equivalence

$$
\operatorname{hocolim}_{w \in \mathcal{C}_{G}(W, d)} G \times_{d w}\left(G / G^{\prime}\right)^{d^{\prime} w} \simeq G \times_{G^{\prime}} E_{G^{\prime}}\left(W_{d^{\prime}, G^{\prime}}, d_{G^{\prime}}\right) .
$$

Proof. Let

$$
R_{w}=\operatorname{Mor}_{\mathcal{C}_{G}(W, d)}(-, w)=\bigsqcup_{[g] \in G / d w} \operatorname{Mor}_{W}(-, g w)=G \times \times_{d w} \operatorname{Mor}_{W}(-, w),
$$

where $\bigsqcup_{[g] \in G / d w} \operatorname{Mor}_{W}(-, g w)$ is topologized as a subspace of $G / d w$. Then for every functor $T: \mathcal{C}_{G}(W, d) \rightarrow G$-CW, $R_{w} \times \mathcal{C}_{G}(W, d) T=T(w)$.

Hence

$$
\begin{aligned}
R_{w} \times \times_{\mathcal{C}_{G}(W, d)} G \times_{d(-)} & \left(G / G^{\prime}\right)^{d^{\prime}(-)}=G \times \times_{d w}\left(G / G^{\prime}\right)^{d^{\prime} w} \\
& =G \times_{d w}\left(\left\{g \in G: d^{\prime} g w \subseteq G^{\prime}\right\} / G^{\prime}\right)=G \times_{G^{\prime}} Y
\end{aligned}
$$

where $Y=\left\{g: d^{\prime} g w \subseteq G^{\prime}\right\} / d w$ is a $G^{\prime}$-subspace of $G / d w$.

We will consider $\mathcal{C}_{G^{\prime}}\left(W_{d^{\prime}, G^{\prime}}, d_{G^{\prime}}\right)$ as a subcategory of $\mathcal{C}_{G}(W, d)$. Then

$$
Y=R_{w} \times_{\mathcal{C}_{G^{\prime}}\left(W_{d^{\prime}, G^{\prime}}, d_{G^{\prime}}\right)} G^{\prime} / d_{G^{\prime}}(-)
$$


and $R_{w}$ after restriction to $\mathcal{C}_{G^{\prime}}\left(W_{d^{\prime}, G^{\prime}}, d_{G^{\prime}}\right)$ is equal to

$$
\bigsqcup_{[g d w] \in Y / G^{\prime}} \operatorname{Mor}_{\mathcal{C}_{G^{\prime}}\left(W_{d^{\prime}, G^{\prime}}, d_{G^{\prime}}\right)}(-, g w) .
$$

Let $E_{d}=B\left(-\downarrow \mathcal{C}_{G}(W, d)\right)$. Then $E_{d}$ is a $\mathcal{C}_{G}(W, d)$-CW-complex whose orbits have the form $R_{w}$. Hence,

$$
\begin{aligned}
\operatorname{hocolim}_{w \in \mathcal{C}_{G}(W, d)} G \times_{d w}\left(G / G^{\prime}\right)^{d^{\prime} w} & =E_{d} \times_{\mathcal{C}_{G}(W, d)} G \times_{d(-)}\left(G / G^{\prime}\right)^{d^{\prime}(-)} \\
& =G \times_{G^{\prime}}\left(E_{d} \times_{\mathcal{C}_{G^{\prime}}\left(W_{d^{\prime}, G^{\prime}}, d_{G^{\prime}}\right)} G^{\prime} / d_{G^{\prime}}(-)\right) .
\end{aligned}
$$

The functor $E_{d}$ after restriction to the category $\mathcal{C}_{G^{\prime}}\left(W_{d^{\prime}, G^{\prime}}, d_{G^{\prime}}\right)$ remains free in the sense of [DF1]. Hence there exists a $G^{\prime}$-homotopy equivalence

$$
E_{d} \times_{\mathcal{C}_{G^{\prime}}\left(W_{d^{\prime}, G^{\prime}}, d_{G}^{\prime}\right)} G^{\prime} / d_{G^{\prime}}(-) \simeq \operatorname{hocolim}_{w \in \mathcal{C}_{G^{\prime}}\left(W_{d^{\prime}, G^{\prime}}, d_{G^{\prime}}\right)} G^{\prime} / d_{G^{\prime}} w .
$$

3.5. Proposition. Suppose that, for every $G^{\prime} \in \mathcal{S}, W_{d^{\prime}, G^{\prime}} / G^{\prime}$ is a discrete space and the map

$$
\operatorname{hocolim}_{w \in \mathcal{C}_{G^{\prime}}\left(W_{d^{\prime}, G^{\prime}}, d_{G^{\prime}}\right)} K / d_{G^{\prime}} w \rightarrow K / G^{\prime}
$$

is a homotopy equivalence. Then:

(i) The map

$$
\operatorname{hocolim}_{w \in \mathcal{C}_{G}(W, d)} K \times_{d w} X^{d^{\prime} w} \rightarrow K \times_{G} X
$$

is a homotopy equivalence if $X$ is a $G$-CW-complex and the isotropy groups of $X$ are in $\mathcal{S}$.

(ii) The $G$-poset $\left\{\left(d w, d^{\prime} w\right): w \in W\right\}$ is $(\mathcal{S}, K)$-essential.

Proof. Let $F_{d^{\prime}}: G-\mathrm{CW} \rightarrow G$-CW be a functor such that

$$
F_{d^{\prime}}(X)=\operatorname{hocolim}_{w \in \mathcal{C}_{G}(W, d)} G \times_{d w} X^{d^{\prime} w} .
$$

It follows from 3.4 that, for every $G^{\prime} \in \mathcal{S}$, there are homotopy equivalences

$$
\begin{aligned}
& K \times_{G} F_{d^{\prime}}\left(G / G^{\prime}\right) \simeq K \times_{G^{\prime}} \operatorname{hocolim}_{w \in \mathcal{C}_{G^{\prime}}\left(W_{d^{\prime}, G^{\prime}}, d_{G^{\prime}}\right)} G^{\prime} / d w \cap G^{\prime} \\
& \quad=\operatorname{hocolim}_{w \in \mathcal{C}_{G^{\prime}}\left(W_{d^{\prime}, G^{\prime}}, d_{G^{\prime}}\right)} K / d_{G^{\prime}} w \simeq K / G^{\prime}=K \times_{G} G / G^{\prime} .
\end{aligned}
$$

Now, it is sufficient to apply 2.2(i) and 3.1.

We now describe some special cases of 3.5.

3.6. ExAmples. (i) Let $W$ be a topological $G$-poset satisfying the condition that $w \leq g w$ implies $w=g w$. Assume that the spaces $d_{n} W / G$ are discrete. Let $d_{s}:$ sd $W \rightarrow \mathcal{S}(G)$ be an admissible function such that

$$
d_{s} w .=G_{w .}=G_{w_{0}} \cap \ldots \cap G_{w_{n}} .
$$

The natural projection $\operatorname{sd} W \rightarrow(\operatorname{sd} W) / G$ induces a natural equivalence of categories $\mathcal{C}_{G}\left(\operatorname{sd} W, d_{s}\right) \rightarrow(\operatorname{sd} W) / G$. It follows from the definitions that 
there is a $G$-homotopy equivalence

$$
\operatorname{hocolim}_{\mathcal{C}_{G}\left(\operatorname{sd} W, d_{s}\right)} G / d_{s}(-) \rightarrow B W .
$$

If $W$ is a $G$-subset of $\mathcal{S}(G)$, then $d_{s}\left(H_{0}, \ldots, H_{n}\right)=N H_{0} \cap \ldots \cap N H_{n}$ and $d^{\prime}\left(H_{0}, \ldots, H_{n}\right)=H_{n}$. Hence 3.5 can be considered as a generalization of 2.3 .

(ii) Let $W$ be a $G$-subposet of $\mathcal{S}(G)$. Then $G_{w}=N_{G} w=N w$. If $d: W^{\mathrm{op}} \rightarrow \mathcal{S}(G)$ is an arbitrary admissible function, then we can take $d^{\prime} w=w$ whenever $w \in W$. Let $d_{c}: W^{\text {op }} \rightarrow \mathcal{S}(G)$ be an admissible map such that, for every $w \in W, d_{c} w=C_{G} w=C w$. Then $\mathcal{C}_{G}\left(W^{\text {op }}, d_{c}\right)=\mathcal{C}_{W}$ is the category whose objects are elements of $W$ and whose morphisms are the group homomorphisms which are restrictions of inner automorphisms of $G$. Let $X$ be a $G$-CW-complex such that all its isotropy groups are compact. If the space hocolim $w_{w \in \mathcal{C}_{W_{H}}} H / C_{H} w$ is $H$-contractible whenever $H$ is an isotropy group of $X$, then the map

$$
\operatorname{hocolim}_{w \in \mathcal{C}_{W}} G \times_{C_{G} w} X^{w} \rightarrow X
$$

is a $G$-homotopy equivalence. If the map

$$
\operatorname{hocolim}_{w \in \mathcal{C}_{W_{H}}} K / C_{H} w \rightarrow K / H
$$

is a homotopy equivalence whenever $H$ is an isotropy group of $X$, then the map

$$
\operatorname{hocolim}_{w \in \mathcal{C}_{W}} K \times_{C_{G} w} X^{w} \rightarrow K \times_{G} X
$$

is also a homotopy equivalence.

(iii) Let $W=\mathcal{A}_{p}(G)$. Then $\mathcal{C}_{G}\left(\mathcal{A}_{p}(G)^{\mathrm{op}}, d_{c}\right)=A_{p}(G)$. If $H$ is a compact Lie group with a non-trivial $p$-subgroup, then there is an $H$-homotopy equivalence

$$
\operatorname{hocolim}_{E \in A_{p}(H)} H / C_{H} E \simeq \mathcal{E} \mathcal{O}_{\mathcal{Z}_{p}(H)}
$$

where $\mathcal{Z}_{p}(G)$ is the poset of all compact subgroups of $G$ with a non-trivial central $p$-subgroup and

$$
\mathcal{E} \mathcal{O}_{\mathcal{Z}_{p}(H)}=E_{H}\left(\mathcal{Z}_{p}(H), \mathrm{id}\right)=\operatorname{hocolim}_{H / H^{\prime} \in \mathcal{O}_{\mathcal{Z}_{p}(H)}} H / H^{\prime} .
$$

Indeed, for every $H^{\prime} \in \mathcal{Z}_{p}(H)$, the space $\left(\operatorname{hocolim}_{E \in A_{p}(H)} H / C_{H} E\right)^{H^{\prime}}=$ $B\left(H / H^{\prime} \downarrow O_{d_{c}}\right)$ is homotopy equivalent to $B\left(H^{\prime} \downarrow d_{c}\right)=B\left(\mathcal{A}_{p}\left(C_{H} H^{\prime}\right)\right)$ and hence is contractible. This implies that there is a $G$-homotopy equivalence

$$
\operatorname{hocolim}_{E \in A_{p}(G)} G \times_{C_{G} E} X^{E} \simeq X
$$

whenever all isotropy groups of $X$ are in $\mathcal{Z}_{p}(G)$.

3.7. ExAmple. Let $V$ be a $G$-subset of $\mathcal{S}(G)$ such that $\mathcal{W}(V) / G$ is discrete. Let

$$
r_{V}(X)=\operatorname{hocolim}_{\left(H, H^{\prime}\right) \in \mathcal{C}(V)} G \times_{H} X^{H^{\prime}} .
$$

This construction is natural in $X$ and $\mathcal{S}\left(G, r_{V}(X)\right) \subseteq V$. The $G$-maps $G \times_{H}$ $X^{H^{\prime}} \rightarrow X$ define a natural transformation of functors $p_{V}: r_{V} \rightarrow \operatorname{Id}_{G-\mathrm{CW}}$. 
There exists a $G$-homotopy equivalence (natural in $X$ )

$$
r_{V}(X) \rightarrow B\left(\operatorname{Map}_{G}(G / e,-), \mathcal{O}_{V}, \operatorname{Map}_{G}(-, X)\right)
$$

where $B(-,-,-)$ is the bar construction described in Section 3 of $[\mathrm{HV}]$ and in Section 4 of $[\mathrm{Dw}]$.

If $G^{\prime} \in V$, then the map $p_{V}(X)^{G^{\prime}}: r_{V}(X)^{G^{\prime}} \rightarrow X^{G^{\prime}}$ is a homotopy equivalence. Indeed, in this case we have homotopy equivalences $\left.\operatorname{~hocolim}_{\left(H, H^{\prime}\right) \in \mathcal{C}(V)} G \times_{H} X^{H^{\prime}}\right)^{G^{\prime}} \simeq \operatorname{hocolim}_{\left(H, H^{\prime}\right) \in \mathcal{W}\left(V\left(\geq G^{\prime}\right)\right)} X^{H^{\prime}} \simeq X^{G^{\prime}}$. Suppose that all isotropy groups of $X$ are in $V$. Then $p_{V}(X): r_{V}(X) \rightarrow X$ is a $G$-homotopy equivalence and gives us a $G$-homotopy decomposition of $X$

$$
\operatorname{hocolim}_{\left(H, H^{\prime}\right) \in \mathcal{C}(V)} G \times_{H} X^{H^{\prime}} \simeq X
$$

from 0.0(i). If $f: X_{1} \rightarrow X_{2}$ is an equivariant map of $G$-CW-complexes and, for every $H \in V, f^{H}: X_{1}^{H} \rightarrow X_{2}^{H}$ is a homotopy equivalence, then $r_{V}(f)$ is a $G$-homotopy equivalence because, for every $\left(H, H^{\prime}\right) \in \mathcal{W}(V), H$ acts trivially on $X^{H^{\prime}}$. Hence, for every $K, \mathcal{W}(V)$ is $(V, K)$-essential.

It follows from the definitions that $p_{V}(X) / G$ gives us a homotopy decomposition of $X / G$ from 0.0 (ii):

$$
\operatorname{hocolim}_{G / H^{\prime} \in \mathcal{O}_{V}} X^{H^{\prime}} \simeq \operatorname{hocolim}_{\left(H, H^{\prime}\right) \in \mathcal{C}(V)} X^{H^{\prime}} \simeq X / G
$$

and that

$$
\begin{aligned}
\mathcal{E O}_{V} & =E_{G}(V, \mathrm{id})=\operatorname{hocolim}_{G / H \in \mathcal{O}_{V}} G / H \\
& =\operatorname{hocolim}_{\left(H, H^{\prime}\right) \in \mathcal{C}(V)} G / H=r_{V}(*) .
\end{aligned}
$$

Let $G^{\prime}$ be a closed subgroup of $G$ and let $V$ be a $G$-subposet of $\mathcal{S}(G)$ such that the spaces $\mathcal{W}(V) / G$ and $\mathcal{W}\left(V_{G^{\prime}}\right) / G^{\prime}$ are discrete. The following two results are consequences of 3.5 and the fact that $\mathcal{C}(V)=\mathcal{C}_{G}\left(\mathcal{W}(V), d_{\mathcal{W}(G)}\right)$ and $r_{V}(*)=E_{G}\left(\mathcal{W}\left(V_{G}\right), d_{\mathcal{W}(G)}\right)$.

3.8. Corollary. There exists a G-homotopy equivalence

$\operatorname{hocolim}_{\left(H, H^{\prime}\right) \in \mathcal{C}(V)} G \times_{H}\left(G / G^{\prime}\right)^{H^{\prime}} \simeq G \times_{G^{\prime}} E_{G^{\prime}}\left(\mathcal{W}\left(V_{G^{\prime}}\right), d_{\mathcal{W}\left(G^{\prime}\right)}\right)$.

3.9. Corollary. Let $f: X_{1} \rightarrow X_{2}$ be a $G$-cellular map such that, for every $H \in V, f^{H}$ is a homotopy equivalence.

(i) If, for every isotropy group $G^{\prime}$ of $X_{i}$, the map $r_{V_{G^{\prime}}}(*) \rightarrow *$ is a $G^{\prime}$-homotopy equivalence, then the maps

$$
\operatorname{hocolim}_{\left(H, H^{\prime}\right) \in \mathcal{C}(V)} G \times_{H} X_{i}^{H^{\prime}} \rightarrow X_{i}
$$

and $f$ are $G$-homotopy equivalences.

(ii) If, for every isotropy group $G^{\prime}$ of $X_{i}$, the map $K \times{ }_{G^{\prime}} r_{V_{G^{\prime}}}(*) \rightarrow K / G^{\prime}$ is a homotopy equivalence, then the maps

$$
\operatorname{hocolim}_{\left(H, H^{\prime}\right) \in \mathcal{C}(V)} K \times_{H} X_{i}^{H^{\prime}} \rightarrow K \times_{G} X_{i}
$$

and $\operatorname{id}_{K} \times_{G} f$ are also homotopy equivalences. 
3.10. ExAmpLe. Let $X$ be a $G$-CW-complex. It follows from 3.6(iii) and 2.5 (iv) that there are $G$-homotopy equivalences

$$
\begin{gathered}
r_{\mathcal{Z}_{p}(G)}(X) \simeq \operatorname{hocolim}_{E \in A_{p}(G)} G \times_{C_{G} E} X^{E}, \\
r_{\mathcal{N}_{p}(G)}(X) \simeq \operatorname{hocolim}_{\left[\left(E_{0}, \ldots, E_{n}\right)\right] \in \operatorname{sd} \mathcal{A}_{p}(G) / G} G \times_{N E_{0} \cap \ldots \cap N E_{n}} X^{E_{n}} .
\end{gathered}
$$

3.11. ExAmple. Let $G$ be a discrete group. Let $V$ be a $G$-poset of subgroups of $G$ satisfying the condition that $v \leq g v$ implies $v=g v$. Let $d: V^{\mathrm{op}} \rightarrow \mathcal{S}(G)$ be an admissible function. It is proved in [JS] that, for every admissible function $d^{\prime \prime}: W \rightarrow \mathcal{S}(G)$, there exists a natural $G$-map $E_{G}\left(W, d^{\prime \prime}\right) \rightarrow B W$ which is a homotopy equivalence. This implies that if, for every isotropy group $G^{\prime}$ of $X$, the space $B V_{\leq G^{\prime}}$ is contractible, then the $G$-maps

$$
\begin{aligned}
\operatorname{hocolim}_{\left(H, H^{\prime}\right) \in \mathcal{C}(V)} G \times_{H} X^{H^{\prime}} & \rightarrow X, \\
\operatorname{hocolim}_{H \in \mathcal{C}_{G}\left(V^{\mathrm{op}, d}\right)} G \times_{d H} X^{H} & \rightarrow X
\end{aligned}
$$

are homotopy equivalences and that, for every free $G$-CW complex $K$, we have homotopy decompositions

$$
\begin{aligned}
\operatorname{hocolim}_{\left(H, H^{\prime}\right) \in \mathcal{C}(V)} K \times_{H} X^{H^{\prime}} & \simeq K \times_{G} X, \\
\operatorname{hocolim}_{H \in \mathcal{C}_{G}\left(V^{\mathrm{op}}, d\right)} K \times_{d H} X^{H} & \simeq K \times_{G} X .
\end{aligned}
$$

Here $V_{\leq G^{\prime}}=\left\{H \in V: H \leq G^{\prime}\right\}$.

3.12. REMARK. One can generalize the above result of [JS] and construct $G$-maps (natural in $X$ )

$$
\begin{aligned}
\operatorname{hocolim}_{\left(H, H^{\prime}\right) \in \mathcal{C}(V)} G \times_{H} X^{H^{\prime}} & \rightarrow Y, \\
\operatorname{hocolim}_{H \in \mathcal{C}_{G}\left(V^{\mathrm{op}, d)}\right.} G \times_{d H} X^{H} & \rightarrow Y,
\end{aligned}
$$

where

$$
Y=\operatorname{hocolim}_{\left[\left(H_{0}, \ldots, H_{n}\right)\right] \in \operatorname{sd} V / G} G \times_{N H_{0} \cap \ldots \cap N H_{n}} X^{H_{n}},
$$

which are homotopy equivalences. Hence, for every free $G$-CW-complex $K$, we have homotopy equivalences

$$
\begin{aligned}
K \times_{G} r_{V}(X) & \simeq \operatorname{hocolim}_{\left[\left(H_{0}, \ldots, H_{n}\right)\right] \in \operatorname{sd} V / G} K \times_{N H_{0} \cap \ldots \cap N H_{n}} X^{H_{n}}, \\
\operatorname{hocolim}_{H \in \mathcal{C}_{G}\left(V^{\mathrm{op}}, d\right)} K \times_{d H} X^{H} & \simeq \operatorname{hocolim}_{\left[\left(H_{0}, \ldots, H_{n}\right)\right] \in \operatorname{sd} V / G} K \times_{N H_{0} \cap \ldots \cap N H_{n}} X^{H_{n}} .
\end{aligned}
$$

4. $h_{G}^{*}$-decompositions of $G$-CW-complexes. Let $G$ be a Lie group and let $h_{G}^{*}$ be a generalized $G$-cohomology theory. Let $h \mathcal{O}_{G}$ be the category whose objects are the same as the objects of $\mathcal{O}_{G}$ and whose morphisms are the $G$-homotopy classes of the morphisms of $\mathcal{O}_{G}$. Let $M$ be a functor from the category $h \mathcal{O}_{G}^{\text {op }}$ to the category $\mathrm{Ab}$ of abelian groups. The ordinary equivariant cohomology of a $G$-CW-complex $Y$ with coefficients in $M$ will 
be denoted by $H_{G}^{*}(Y, M)$. These cohomology groups, in the case when $G$ is a finite group, was defined in [Br1]. The case of a Lie group is described in [Wi] and in the appendix of [JMO]. For any generalized $G$-cohomology theory $h_{G}^{*}$ on $G$-CW, there is a spectral sequence

$$
H_{G}^{m}\left(Y, h_{G}^{n}(-)\right) \Rightarrow h_{G}^{m+n}(Y) .
$$

For every closed subgroup $H$ of $G$, the $H$-cohomology theory such that $h_{H}^{*}\left(X^{\prime}\right)=h_{G}^{*}\left(G \times_{H} X^{\prime}\right)$ whenever $X^{\prime}$ is an $H$-CW-complex will be denoted by $h_{H}^{*}$. This gives us a functor $h_{H(-)}^{*}\left(X^{H^{\prime}(-)}\right)$ defined on the homotopy category $h \mathcal{C}$ associated to $\mathcal{C}$. This functor can be considered as coefficients of the generalized cohomology theory $h_{G}^{*}\left(-\times_{\mathcal{C}}\left(G \times_{H(-)} X^{H^{\prime}(-)}\right)\right)$ defined on the category of free $\mathcal{C}$-CW-complexes in the sense of [DF1], i.e. $\mathcal{C}$-CW-complexes with orbits of the form $\operatorname{Mor}_{\mathcal{C}}(-, c)$. For every contravariant functor $M: h \mathcal{C} \rightarrow \mathrm{Ab}, H^{*}(\mathcal{C}, M)=\operatorname{Tor}_{\mathcal{C}}^{*}(\mathbb{Z}, M)$ is equal to the Bredon cohomology groups $H_{\mathcal{C}}^{*}(B(-\downarrow \mathcal{C}), M)$ (Sections 4 and 5 of [DF1]). Recall that

$$
\operatorname{hocolim}_{c \in \mathcal{C}}\left(G \times_{H}(-) X^{H^{\prime}(-)}\right)=B(-\downarrow \mathcal{C}) \times_{\mathcal{C}} G \times_{H(-)} X^{H^{\prime}(-)} .
$$

Let $W$ be a $G$-subposet of $\mathcal{W}(G)$. Let $F=\left(H(-), H^{\prime}(-)\right): \mathcal{C} \rightarrow$ $\mathcal{C}_{G}\left(W, d_{\mathcal{W}(G)}\right)$ be a functor such that the map

$$
p_{F}(X): \operatorname{hocolim}_{c \in \mathcal{C}} G \times_{H(c)} X^{H^{\prime}(c)} \rightarrow X
$$

is an $h_{G}^{*}$-decomposition of $X$, i.e. the map

$$
h_{G}^{*}(X) \rightarrow h_{G}^{*}\left(\operatorname{hocolim}_{c \in \mathcal{C}} G \times_{H(c)} X^{H^{\prime}(c)}\right)
$$

is an isomorphism. It follows from 5.3 of [DF1] that there exists a spectral sequence

$$
H^{m}\left(\mathcal{C}, h_{H(-)}^{n}\left(X^{H^{\prime}(-)}\right)\right) \Rightarrow h_{G}^{m+n}(X) .
$$

The results of this section describe and use this spectral sequence in many examples.

We remark that if $X=*$ and $F=G / H(-): \mathcal{C} \rightarrow \mathcal{O}_{G}$, then we obtain the spectral sequence of the generalized cohomology theory $h_{G}^{*}$ on $Y=$ $\operatorname{hocolim}_{c \in \mathcal{C}} F(c)$.

Let $f: X_{1} \rightarrow X_{2}$ be a $G$-CW-complex map and let $p_{F}\left(X_{i}\right)$ be an $h_{G}^{*}$ decomposition of $X_{i}$ for $i=1,2$. If, for every $c \in \mathcal{C}, h_{H(c)}^{*}\left(X_{2}^{H^{\prime}(c)}\right) \rightarrow$ $h_{H(c)}^{*}\left(X_{1}^{H^{\prime}(c)}\right)$ is an isomorphism then the map $h^{*}(f): h_{G}^{*}\left(X_{2}\right) \rightarrow h_{G}^{*}\left(X_{1}\right)$ is an isomorphism. This motivates the following definition.

4.0. Definition. Let $\mathcal{S}$ be a $G$-subposet of $\mathcal{S}(G)$. Let $W$ be a $G$-subposet of $\mathcal{W}(G)$. We will say that $W$ is $\left(\mathcal{S}, h_{G}^{*}\right)$-essential if, for every equivariant cellular map $f: X \rightarrow Y$ of $G$-CW-complexes whose isotropy groups 
are all in $\mathcal{S}$, the condition that $h_{H}^{*}\left(Y^{H^{\prime}}\right) \rightarrow h_{H}^{*}\left(X^{H^{\prime}}\right)$ is an isomorphism whenever $\left(H, H^{\prime}\right) \in W$ implies that $h_{G}^{*}(Y) \rightarrow h_{G}^{*}(X)$ is an isomorphism.

In particular, if $W$ is $\left(\mathcal{S}_{0}(G, X), h_{G}^{*}\right)$-essential and $h_{H}^{*}(*) \rightarrow h_{H}^{*}\left(X^{H^{\prime}}\right)$ is an isomorphism whenever $\left(H^{\prime}, H\right) \in W$, then $h_{G}^{*}(*) \rightarrow h_{G}^{*}(X)$ is an isomorphism.

The following result can be used to construct many non-trivial examples of $h_{G}^{*}$-essential posets.

4.1. Proposition. Let $F=\left(H(-), H^{\prime}(-)\right): \mathcal{C} \rightarrow \mathcal{C}_{G}\left(W, d_{\mathcal{W}(G)}\right)$ be a functor such that for every $G^{\prime} \in \mathcal{S}$, the map

$$
\operatorname{hocolim}_{c \in \mathcal{C}} G \times_{H(c)}\left(G / G^{\prime}\right)^{H^{\prime}(c)} \rightarrow G / G^{\prime}
$$

is an $h_{G}^{*}$-equivalence. Then:

(i) The map $p_{F}(X)$ is an $h_{G}^{*}$-decomposition of $X$ if all isotropy groups of $X$ are in $\mathcal{S}$.

(ii) The poset $W$ is $\left(\mathcal{S}, h_{G}^{*}\right)$-essential.

Proof. Let

$$
h_{G}^{\prime *}(X)=h_{G}^{*}\left(\operatorname{hocolim}_{c \in \mathcal{C}} G \times_{H(c)} X^{H^{\prime}(c)}\right) .
$$

Then $p_{F}$ induces a natural transformation $p^{*}: h_{G}^{*} \rightarrow h_{G}^{*}$ of $G$-cohomology theories. If the assumption of the proposition holds, then $p^{*}(X)$ is an isomorphism. Hence $p_{F}(X)$ is an $h_{G}^{*}$-equivalence.

Let $R$ be a commutative ring. The generalized $G$-cohomology theories from the category $G$-CW to the category $R^{*}$-Mod of graded $R$-modules will be called $R$-G-cohomology theories.

Let $V$ be a $G$-poset of compact subgroups of $G$. Recall that $\mathcal{C}(V)$ is a full subcategory of $\mathcal{C}_{G}$ whose objects are the elements of the poset $\mathcal{W}(V)$ of pairs $\left(H, H^{\prime}\right)$ such that $H$ is a subgroup of $H^{\prime}$ and $H^{\prime} \in V$.

4.2. Proposition. Let $h_{G}^{*}=\left\{h^{n}\right\}_{n \in \mathbb{N}}$ be an $R$-G-cohomology theory. Let $\mathcal{S}$ and $V$ be $G$-posets of compact subgroups of $G$ such that, for every $H \in \mathcal{S}, h_{H}^{*}(*) \rightarrow h_{H}^{*}\left(r_{V_{H}}(*)\right)$ is an isomorphism. Then:

(i) The $G$-poset $\mathcal{W}(V)$ is $\left(\mathcal{S}, h_{G}^{*}\right)$-essential.

(ii) Let $f: X \rightarrow Y$ be a map of $G$-CW-complexes whose isotropy groups are all in $\mathcal{S}$. If , for every $H \in V$, the map $X^{H} \rightarrow Y^{H}$ is an $R$-equivalence, then $h_{G}^{*}(Y) \rightarrow h_{G}^{*}(X)$ is an isomorphism.

Proof. (i) is a consequence of 3.8 and 4.1(ii).

(ii) Propositions 4.1(i) and 3.8 imply that, for every $G$-CW-complex $X$ whose isotropy groups are in $\mathcal{S}$, there exists a spectral sequence

$$
H^{m}\left(\mathcal{C}(V), h_{H(-)}^{n}\left(X^{H^{\prime}(-)}\right)\right) \Rightarrow h_{G}^{m+n}(X) .
$$


This spectral sequence is natural in $X$. The assumption implies that, for every $\left(H, H^{\prime}\right) \in \mathcal{C}(V)$, the map $h_{H}^{*}\left(Y^{H^{\prime}}\right) \rightarrow h_{H}^{*}\left(X^{H^{\prime}}\right)$ is an isomorphism because $H \subseteq H^{\prime}$. Hence the map $X \rightarrow Y$ is an $h_{G}^{*}$-equivalence.

4.3. Examples. Let $h_{G}^{*}(X)=H^{*}\left(K \times_{G} X, R\right)$. Then $h_{G}^{*}(G / H)=$ $H^{*}(K / H, R)$.

(i) Let $K=*, R=F_{p}$. It is proved in [JMO] $(1.2,2.2,2.12)$ that, if $H$ is a compact Lie group and $\operatorname{dim} H>0$, then the space $\mathcal{E} \mathcal{O}_{\mathcal{T}_{p}(H)}=r_{\mathcal{T}_{p}(H)}(*)$ is $F_{p}$-acyclic. Let $\mathcal{S}_{d}(G)$ denote the set of all compact subgroups $H$ of $G$ such that $\operatorname{dim} H>0$. Let $f: X \rightarrow Y$ be a map of $G$-CW-complexes whose isotropy groups are all in $\mathcal{S}_{d}(G)$. If, for every non-trivial $p$-toral subgroup $H$ of $G$, the map $f^{H}: X^{H} \rightarrow Y^{H}$ is an $F_{p}$-homology isomorphism, then so is $f$. In particular, let $G$ be a compact Lie group. If all isotropy groups of $X$ are in $\mathcal{S}_{d}(G)$ and, for every non-trivial $p$-toral subgroup $H$ of $G, X^{H}$ is $F_{p}$-acyclic, then $X$ is $F_{p}$-acyclic.

(ii) Let $\mathcal{A}_{p}^{\prime}(G)=\mathcal{A}_{p}(G) \cup\{e\}$. If $H \in \mathcal{Z}_{p}(G)$ and $E \in \mathcal{A}_{p}^{\prime}(H)$, then the space $\mathcal{E O}_{\mathcal{A}_{p}(H)} / E=\mathcal{E O}_{\mathcal{A}_{p}^{\prime}(H)} / E$ is contractible. Let $f: X \rightarrow Y$ be a map of $G$-CW-complexes whose isotropy groups are all in $\mathcal{Z}_{p}(G)$. Suppose that, for every $E \in \mathcal{A}_{p}(G), f^{E}$ is an $R$-homology isomorphism and that, for every $k \in K$ and $x \in X \cup Y, G_{x} \cap G_{k}$ is an elementary abelian $p$-subgroup of $G_{x}$. This implies that, for every $x \in X \cup Y$, the map $K \times_{G_{x}} \mathcal{E O}_{\mathcal{A}_{p}\left(G_{x}\right)} \rightarrow K / G_{x}$ is an $R$-homology isomorphism. Hence the map $K \times_{G} X \rightarrow K \times_{G} Y$ is an $R$-homology isomorphism.

(iii) Let $K=*$. Then we obtain 0.3 (ii) as a consequence of 4.2 and 1.4.

Let $h_{G}^{*}(X)=H^{*}\left(K \times_{G} X, F_{p}\right)$. In this case there is a spectral sequence

$$
H_{G}^{m}\left(K, H^{n}\left(X \times_{G}(-), F_{p}\right)\right) \Rightarrow h_{G}^{n+m}(X) .
$$

Hence if, for all maximal $p$-toral subgroups $P$ of isotropy groups of $K, X / P$

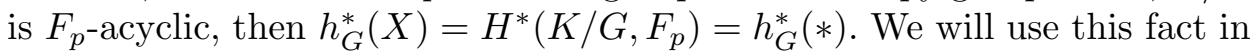
the following examples.

4.4. ExAmples. Let $h_{G}^{*}=H^{*}\left(K \times_{G}-, F_{p}\right)$. Let $f: X \rightarrow Y$ be an equivariant cellular map of $G$-CW-complexes with compact isotropy groups.

(i) Let $S_{p}^{\prime}(G)$ be the poset of all subgroups of $p$-toral subgroups of $G$, and let $S_{p}(G)$ be the subposet of $S_{p}^{\prime}(G)$ consisting of all subgroups which contain a non-trivial $p$-subgroup. Let $H$ be a compact subgroup of $G$. Then $h_{H}^{*}(*)=$ $h_{H}^{*}\left(r_{S_{p}^{\prime}(H)}(*)\right)$ because, for every $p$-toral subgroup $P$ of $H, r_{S_{p}^{\prime}(H)}(*) / H$ is $F_{p}$-acyclic. It follows from Section 3 of [JO] that the maps $H_{H}^{m}\left(*, h_{H}^{n}\right) \rightarrow$ $H_{H}^{m}\left(r_{S_{p}^{\prime}(H)}(*), h_{H}^{n}\right)$, where $m>0$, are isomorphisms. Hence so are the maps $H_{H}^{0}\left(*, h_{H}^{n}\right) \rightarrow H_{H}^{0}\left(r_{S_{p}^{\prime}(H)}(*), h_{H}^{n}\right)$. From 3.3 of [JO] and 1.2 and 2.2 of [JMO], it follows that the map $r_{\mathcal{T}_{p}^{\prime}(H)}(*) \rightarrow r_{S_{p}^{\prime}(H)}(*)$ induces isomorphisms in $h_{H}^{*}$ and $H_{H}^{*}\left(-, h_{H}^{n}\right)$. This implies that the maps $h_{G}^{*}(Y) \rightarrow h_{G}^{*}(X)$ and 
$H_{G}^{*}\left(Y, h_{G}^{n}\right) \rightarrow H_{G}^{*}\left(X, h_{G}^{n}\right)$ are isomorphisms if, for every $p$-toral subgroup $P$ of $G, f^{P}$ is a mod $p$ homology isomorphism.

(ii) Suppose that, for all $n>0, H^{n}\left(K, F_{p}\right)=0$. Let $n>0$. In this case $h_{H}^{n}(H / e)=0$ and (i) implies that the $\operatorname{map} H_{H}^{*}\left(*, h_{H}^{n}\right) \rightarrow H_{H}^{*}\left(r_{\mathcal{T}_{p}(H)}(*), h_{H}^{n}\right)$ is an isomorphism. Hence $H_{G}^{*}\left(Y, h_{G}^{n}\right) \rightarrow H_{G}^{*}\left(X, h_{G}^{n}\right)$ is an isomorphism if, for every non-trivial $p$-toral subgroup $P$ of $G, f^{P}$ is a mod $p$ homology isomorphism.

(iii) Suppose that $K$ is $F_{p^{-}}$-acyclic. Then, for every $H / H^{\prime} \in \mathcal{O}_{H}, h_{H}^{0}\left(H / H^{\prime}\right)$ $=F_{p}$ and $h_{H}^{0}(-)$ is the constant functor after restriction to $\mathcal{O}_{H}$. It follows from 1.2 and 2.2 of [JMO] and Proposition 2 and Theorem 3 of [O1] that the map

$$
H^{*}\left(r_{\mathcal{T}_{p}}(H)(*) / H, F_{p}\right) \rightarrow H^{*}\left(r_{\mathcal{T}_{p}^{\prime}(H)}(*) / H, F_{p}\right)
$$

is an isomorphism. By (ii), so is $h_{H}^{*}\left(r_{\mathcal{T}_{p}(H)}\right) \rightarrow h_{H}^{*}\left(r_{\mathcal{T}_{p}^{\prime}(H)}(*)\right)$. Suppose that all isotropy groups of $X$ and $Y$ contain non-trivial $p$-subgroups. If, for every non-trivial $p$-toral subgroup $P$ of $G, f^{P}$ is a mod $p$ homology isomorphism, then, for all natural $n$, the maps $H_{G}^{*}\left(Y, h_{G}^{n}\right) \rightarrow H_{G}^{*}\left(X, h_{G}^{n}\right)$ and $H^{n}\left(K \times_{G} Y, F_{p}\right) \rightarrow H^{n}\left(K \times_{G} X, F_{p}\right)$ are isomorphisms. In particular, we obtain 0.4. If $G$ is a compact Lie group, then we can take $X=B \mathcal{A}_{p}(G)$, $Y=*$ (cf. the proof of 1.5 ) to obtain 0.5.

(iv) Let $K$ be a $G$-CW-complex such that, for every $k \in K$ and for every $p$-toral subgroup $P$ of $G_{x}, G_{k} \cap P$ is an elementary abelian $p$-group. Suppose that $K$ is $F_{p}$-acyclic. (In particular, we can take $K=E G$.) If all isotropy groups of $X$ and $Y$ contain non-trivial $p$-subgroups and, for every $E \in \mathcal{A}_{p}(G), f^{E}$ is a mod $p$ homology isomorphism, then $K \times{ }_{G} X \rightarrow$ $K \times_{G} Y$ is a mod $p$ homology isomorphism. Indeed, it follows from 4.3(ii) that $K \times{ }_{G} r_{\mathcal{T}_{p}(G)}(X) \rightarrow K \times{ }_{G} r_{\mathcal{T}_{p}(G)}(Y)$ is a $\bmod p$ homology isomorphism. Now we can use the fact that, by (iii), $K \times_{G} r_{\mathcal{T}_{p}(G)}(X) \rightarrow K \times_{G} X$ is a mod $p$ homology isomorphism.

4.5. ExAmples. Let $G$ be a discrete group and $A$ a $\mathbb{Z}(G)$-module. We will consider the Bredon cohomology theory $h_{G}^{*}=H_{G}^{*}\left(-, M_{A}\right)$, where $M_{A}(-)$ $=\operatorname{Hom}_{\mathbb{Z}(G)}(\mathbb{Z}(-), A)$. Hence

$$
H_{G}^{n}\left(X, M_{A}\right)=H^{n}\left(\operatorname{Hom}_{\mathbb{Z}(G)}\left(C_{*}(X), A\right)\right)
$$

where $C_{*}(X)$ is the ordinary cellular chain complex of $X$. For every $G / H \in$ $\mathcal{O}_{G}$, we have $h_{G}^{*}(G / H)=M_{A}(G / H)=A^{H}$.

(i) Let $G$ be a finite group. Suppose that there is a non-trivial $p$-subgroup $P$ of $G$ such that every element of $P$ acts trivially on $A$. Then

$$
H_{G}^{*}\left(\left|\mathcal{S}_{p}(G)\right|, M_{A}\right)=A^{G}=H_{G}^{*}\left(*, M_{A}\right) .
$$

Indeed, $M_{A}$ is a Hecke functor and it follows from the results of [Wa1] that if $A$ is an $R(G)$-module and, for every subgroup $H$ of $G, X / H$ is $R$ acyclic, then $H_{G}^{*}\left(X, M_{A}\right)=A^{G}$. Let $H$ be a normal subgroup of $G$ with 
a non-trivial $p$-subgroup and let $G^{\prime}=G / H$. If $A^{\prime}$ is a $\mathbb{Z}\left(G^{\prime}\right)$-module, then $H_{G^{\prime}}^{*}\left(\left|\mathcal{S}_{p}(G)\right| / H, M_{A^{\prime}}\right)=A^{\prime G^{\prime}}$ because, by $2.8,\left|\mathcal{S}_{p}(G)\right| / H^{\prime}$ is contractible whenever $H \subseteq H^{\prime} \subseteq G$.

(ii) Let $G$ be a discrete group. Let $\mathcal{S}_{A}(G)$ denote the set of all finite subgroups $H$ of $G$ with a non-trivial $p$-subgroup $P$ such that every element of $P$ acts as identity on $A$. Suppose that all isotropy groups of $X$ and $Y$ are in $\mathcal{S}_{A}(G)$. The map $H_{G}^{*}\left(Y, M_{A}\right) \rightarrow H_{G}^{*}\left(X, M_{A}\right)$ is an isomorphism if, for every compact subgroup $H$ of $G$ with a non-trivial normal $p$-toral subgroup, $f^{H}$ is a homology isomorphism.

(iii) Let $A$ be an $F_{p}(G)$-module. Let $K$ be a $G$-CW-complex. Suppose that all isotropy groups of points of $X$ and $Y$ are finite. In this case the $\operatorname{maps} h_{G}^{*}(K \times Y) \rightarrow h_{G}^{*}(K \times X)$ and

$$
H_{G}^{*}\left(Y, h_{G}^{n}(K \times(-))\right) \rightarrow H_{G}^{*}\left(X, h_{G}^{n}(K \times(-))\right)
$$

are isomorphisms if, for every $p$-subgroup $P$ of $G, f^{P}$ is a $\bmod p$ homology isomorphism. This is a consequence of the fact that, for every Hecke functor $M: \mathcal{O}_{G}^{\mathrm{op}} \rightarrow F_{p^{-}}$Mod, $M(G / G)=H_{G}^{*}\left(r_{\mathcal{T}_{p}^{\prime}(G)}, M\right)$ (1.29 of $\left.[\mathrm{S} 3]\right)$.

Let $W$ be a topological $G$-poset satisfying the condition that $w \leq g w$, where $g \in G$, implies that $w=g w$. Let $d: W \rightarrow \mathcal{S}(G)$ be an admissible function and let $d^{\prime}: W^{\text {op }} \rightarrow \mathcal{S}(G)$ be a $G$-poset map. The next result follows immediately from 3.4 and 4.1 .

4.6. Proposition. Suppose that, for every isotropy group $H$ of the action of $G$ on $X$, the space $W_{d^{\prime}, H} / H$ is discrete and the map

$$
h_{H}^{*}(*) \rightarrow h_{H}^{*}\left(\operatorname{hocolim}_{w \in \mathcal{C}_{H}\left(W_{d^{\prime}, H}, d_{H}\right)} H / H \cap d w\right)
$$

is an isomorphism. Then so is the map

$$
h_{G}^{*}(X) \rightarrow h_{G}^{*}\left(\operatorname{hocolim}_{w \in \mathcal{C}_{G}(W, d)} G \times_{d w} X^{d^{\prime} w}\right)
$$

and there is a spectral sequence

$$
H^{m}\left(\mathcal{C}_{G}(W, d), h_{d(-)}^{n}\left(X^{d^{\prime}(-)}\right)\right) \Rightarrow h_{G}^{m+n}(X) .
$$

4.7. Example. Let $K$ be a $G$-CW-complex. Suppose that, for every $x \in X$, the map

$$
H^{*}\left(K / G_{x}, R\right) \rightarrow H^{*}\left(\operatorname{hocolim}_{w \in \mathcal{C}_{G_{x}}\left(W_{d^{\prime}, G_{x}}, d_{G_{x}}\right)} K / G_{x} \cap d w, R\right)
$$

is an isomorphism. Then so is the map

$$
H^{*}\left(K \times_{G} X, R\right) \rightarrow H^{*}\left(\operatorname{hocolim}_{w \in \mathcal{C}_{G}(W, d)} K \times_{d w} X^{d^{\prime} w}, R\right)
$$

and there is a spectral sequence

$$
H^{m}\left(\mathcal{C}_{G}(W, d), H^{n}\left(K \times_{d(-)} X^{d^{\prime}(-)}, R\right)\right) \Rightarrow H^{m+n}\left(K \times_{G} X, R\right) .
$$


In particular, if, for every isotropy group $H$ of $X, B \mathcal{C}_{H}\left(W_{d^{\prime}, H}, d_{H}\right)$ is $R$ acyclic, then there is a spectral sequence

$$
H^{m}\left(\mathcal{C}_{G}(W, d), H^{n}\left(X^{d^{\prime} w} / d w, R\right)\right) \Rightarrow H^{m+n}(X / G, R) .
$$

4.8. Examples. Let $W$ be a poset of closed subgroups of $G$ satisfying the condition that $w \leq g w$ implies $w=g w$ and such that the spaces $d_{n} W / G$ are discrete. Let $d: W^{\text {op }} \rightarrow \mathcal{S}(G)$ be an admissible function.

(i) Suppose that the map

$$
h_{H}^{*}(*) \rightarrow h_{H}^{*}\left(\operatorname{hocolim}_{w \in \mathcal{C}_{H}\left(W_{H}^{\mathrm{op}}, d_{H}\right)} H / d w\right)
$$

is an isomorphism whenever $H$ is an isotropy group of $X$. Then the map

$$
h_{G}^{*}(X) \rightarrow h_{G}^{*}\left(\operatorname{hocolim}_{w \in \mathcal{C}_{G}\left(W^{\mathrm{op}}, d\right)} G \times_{d w} X^{w}\right)
$$

is an isomorphism and there is a spectral sequence

$$
H^{m}\left(\mathcal{C}_{G}\left(W^{\mathrm{op}}, d\right), h_{d w}^{n}\left(X^{w}\right)\right) \Rightarrow h_{G}^{m+n}(X) .
$$

(ii) Suppose that the map

$$
h_{H}^{*}(*) \rightarrow h_{H}^{*}\left(B W_{H}\right)
$$

is an isomorphism whenever $H$ is an isotropy group of $X$. Then the map $h_{G}^{*}(X) \rightarrow h_{G}^{*}\left(\operatorname{hocolim}_{[w .] \in \operatorname{sd} W / G} G \times_{G_{w} .} X^{w_{n}}\right)=h_{G}^{*}\left(\operatorname{hocolim}_{w . \in \operatorname{sd} W} X^{w_{n}}\right)$ is an isomorphism and there is a spectral sequence

$$
H^{m}\left(\operatorname{sd} W / G, h_{G_{w}}^{n}\left(X^{w_{n}}\right)\right) \Rightarrow h_{G}^{m+n}(X) .
$$

(iii) Let $G$ be a discrete group. Let $K$ be a free $G$-CW-complex. Suppose that the map

$$
K \times_{H} B W_{H} \rightarrow K / H
$$

is a $\bmod p$ homology isomorphism whenever $H$ is an isotropy group of $X$. Then, similarly to 3.11 , the map

$$
\operatorname{hocolim}_{\mathcal{C}_{G}\left(W^{\text {op }, d)}\right.} K \times_{d w} X^{w} \rightarrow K \times_{G} X
$$

is a $\bmod p$ homology isomorphism.

4.9. Examples. Let $W=\mathcal{A}_{p}(G)$. Let $X$ be a $G$-CW-complex such that all its isotropy groups are compact and contain non-trivial $p$-subgroups. Let $K$ be an $F_{p}$-acyclic $G$-CW-complex.

(i) Let $d=d_{c}$. Then $\mathcal{C}_{G}\left(\mathcal{A}_{p}(G)^{\mathrm{op}}, d_{c}\right)=A_{p}(G)$. Suppose that, for every isotropy group $H$ of the action of $G$ on $X$, the map $h_{H}^{*}(*) \rightarrow h_{H}^{*}\left(\mathcal{E O}_{\mathcal{Z}_{p}(H)}\right)$ is an isomorphism. Then it follows from 3.6(iii) that the map

$$
h_{G}^{*}(X) \rightarrow h_{G}^{*}\left(\operatorname{hocolim}_{E \in A_{p}(G)} G \times_{C_{G} E} X^{E}\right)
$$

is an isomorphism and there is a spectral sequence

$$
H^{m}\left(A_{p}(G), h_{C_{G} E}^{n}\left(X^{E}\right)\right) \Rightarrow h_{G}^{m+n}(X) .
$$


Let $h_{G}^{*}=H_{G}^{*}\left(K \times_{G}-, F_{p}\right)$. It follows from 4.4(iii) that if $H$ is a compact subgroup of $G$ and contains a non-trivial $p$-subgroup, then $h_{H}^{*}(*)=$ $h_{H}^{*}\left(\mathcal{E O}_{\mathcal{Z}_{p}(H)}\right)$. Hence there is a mod $p$ homology isomorphism

$$
\operatorname{hocolim}_{w \in A_{p}(G)} K \times_{C_{G} w} X^{w} \rightarrow K \times_{G} X,
$$

and there exists a spectral sequence

$$
H^{n}\left(A_{p}(G), H^{m}\left(K \times_{C_{G} w} X^{w}, F_{p}\right)\right) \Rightarrow H^{n+m}\left(K \times_{G} X, F_{p}\right) .
$$

If $K=E G$, then we obtain the case investigated in [H1,2].

(ii) The map

$$
\operatorname{hocolim}_{[E .] \in \operatorname{sd}} \mathcal{A}_{p}(G) / G K \times_{G_{E} .} X^{E_{n}} \rightarrow K \times_{G} X
$$

is a $\bmod p$ homology isomorphism and there is a spectral sequence

$$
H^{m}\left(\operatorname{sd} \mathcal{A}_{p}(G) / G, H^{n}\left(K \times_{G_{E} .} X^{E_{n}}, F_{p}\right)\right) \Rightarrow H^{m+n}\left(K \times_{G} X, F_{p}\right) .
$$

In particular, if $\mathcal{A}$ is one of the classes $\mathcal{B}_{k}\left(F_{p}\right)$ or $\mathcal{D}\left(F_{p}\right)$ described in 1.3 and, for every $\left(E_{0}, \ldots, E_{n}\right) \in \operatorname{sd} \mathcal{A}_{p}(G), K \times_{N E_{0} \cap \ldots \cap N E_{n}} X^{E_{n}} \in \mathcal{A}$, then $K \times_{G} X \in \mathcal{A}$.

\section{References}

[Br1] G. E. Bredon, Equivariant Cohomology Theory, Lecture Notes in Math. 34, Springer, 1967.

[Br2] - Introduction to Compact Transformation Groups, Academic Press, New York, 1972.

[BK] A. K. Bousfield and D. M. Kan, Homotopy Limits, Completions and Localizations, Lecture Notes in Math. 304, Springer, Berlin, 1972.

[DF1] E. Dror Farjoun, Homotopy and homology of diagrams of spaces, in: Lecture Notes in Math. 1286, Springer, 93-134.

[DF2] - Cellular Spaces, Null Spaces and Homotopy Localization, Lecture Notes in Math. 1622, Springer, 1995.

[Dw] W. G. Dwyer, Homology approximations for classifying spaces of finite groups, Topology 36 (1997), 783-804.

[DK] W. G. Dwyer and D. M. Kan, A classification theorem for diagrams of simplicial sets, Topology 23 (1984), 139-155.

[E] A. D. Elmendorf, Systems of fixed point sets, Trans. Amer. Math. Soc. 277 (1983), $275-284$.

[H1] H. W. Henn, Centralizers of elementary abelian p-subgroups, the Borel construction of the singular locus and applications to the cohomology of discrete groups, Topology 36 (1997), 271-286.

[H2] - Commutative algebra of unstable K-modules, Lannes T-functor and the equivariant mod-p cohomology, J. Reine Angew. Math. 478 (1996), 189-215.

[HV] J. Hollender and R. M. Vogt, Modules of topological spaces, applications to homotopy limits and $E_{\infty}$ stuctures, Arch. Math. (Basel) 59 (1992), 115-129.

[JM1] S. Jackowski and J. McClure, Homotopy approximations for classifying spaces of compact Lie groups, in: Algebraic Topology (Arcata, 1986), Springer, 1989, 221-234. 
[JM2] S. Jackowski and J. McClure, Homotopy decomposition of classifying spaces via elementary abelian subgroups, Topology 31 (1992), 113-132.

[JMO] S. Jackowski, J. McClure and B. Oliver, Homotopy classification of self-maps of BG via G-actions, Ann. of Math. 135 (1992), 183-270.

[JO] S. Jackowski and B. Oliver, Vector bundles over classifying spaces of compact Lie groups, Acta Math. 176 (1996), 109-143.

[JS] S. Jackowski and J. Słomińska, G-functors, G-posets and homotopy decompositions of $G$-spaces, Fund. Math., to appear.

$[\mathrm{LMM}]$ L. G. Lewis, J. P. May and J. McClure, Ordinary $R O(G)$-graded cohomology, Bull. Amer. Math. Soc. 4 (1981), 208-212.

[LMS] L. G. Lewis, J. P. May and M. Steinberger, Equivariant Stable Homotopy Theory, Lecture Notes in Math. 1213, Springer, 1986.

[O1] B. Oliver, A proof of the Conner conjecture, Ann. of Math. 103 (1976), 637-644.

[O2] - A transfer for compact Lie group actions, Proc. Amer. Math. Soc. 97 (1976), $546-548$.

[Q] D. Quillen, Homotopy properties of the poset of non-trivial p-subgroups of a group, Adv. Math. 28 (1978), 101-128.

[S1] J. Słomińska, Some spectral sequences in Bredon cohomology, Cahiers Topologie Géom. Différentielle Catégoriques 33 (1992), 99-134.

[S2] - Homotopy colimits on E-I-categories, in: Algebraic Topology (Poznań, 1989), Lecture Notes in Math. 1474, Springer, 1991, 273-294.

[S3] - Hecke structure on Bredon cohomology, Fund. Math. 140 (1991), 1-30.

[S4] - Cohomology decompositions of the Borel construction, preprint, Torun, 1996.

[Sy] P. Symonds, The orbit space of the p-subgroup complex is contractible, Comment. Math. Helv. 73 (1998), 400-405.

[TW] J. Thévenaz and P. J. Webb, Homotopy equivalence of posets with a group action, J. Combin. Theory Ser. A 56 (1991), 173-181.

[T] R. W. Thomason, Homotopy colimits in the category of small categories, Proc. Cambridge Philos. Soc. 85 (1979), 91-109.

[Wa1] S. Waner, A generalization of cohomology of groups, Proc. Amer. Math. Soc. 85 (1982), 469-474.

[Wa2] -, Mackey functors and G-cohomology, ibid. 90 (1984), 641-648.

[We] P. J. Webb, A split exact sequences of Mackey functors, Comment. Math. Helv. 66 (1991), 34-69.

[Wi] S. J. Wilson, Equivariant homology theories on G-complexes, Trans. Amer. Math. Soc. 212 (1975), 155-171.

[Ž $\quad$ R. T. Živaljević, Combinatorics of topological posets: homotopy complementation formulas, Adv. Appl. Math. 21 (1998), 547-574.

Faculty of Mathematics and Information Sciences

Technical University of Warsaw

Pl. Politechniki 1

00-661 Warszawa, Poland

E-mail: jolslom@impan.gov.pl

Received 14 April 1999;

in revised form 22 January 2001 